\title{
Heme $b$ in marine phytoplankton and particulate material from the North Atlantic Ocean
}

\author{
David J. Honey ${ }^{1}$, Martha Gledhill ${ }^{1, *}$, Thomas S. Bibby ${ }^{1}$, François-Eric Legiret ${ }^{1}$, \\ Nicola J. Pratt ${ }^{1}$, Anna E. Hickman ${ }^{1}$, Tracy Lawson ${ }^{2}$, Eric P. Achterberg ${ }^{1}$ \\ ${ }^{1}$ Ocean and Earth Science, University of Southampton, National Oceanography Centre-Southampton, Southampton SO14 3ZH, UK \\ ${ }^{2}$ Department of Biological Sciences, University of Essex, Wivenhoe Park, Colchester CO4 3SQ, UK
}

ABSTRACT: Concentrations of heme $b$, the ironcontaining prosthetic group of many hemoproteins, were measured in 6 species of marine phytoplankton (Dunaliella tertiolecta, Emiliania huxleyi, Thalassiosira weissflogii, T. oceanica, Phaeodactylum tricornutum and Synechococcus sp. WH7803) that were subjected to variations in iron concentration. Changes in heme $b$ in response to reduced light and nitrate were also examined for E. huxleyi and T. oceanica. Results from laboratory cultures were compared with heme $b$ determined in particulate material in the North Atlantic. In cultures, heme $b$ made up $18 \pm 14 \%$ (SE) of the total iron pool. Reduced iron and nitrate concentrations resulted in a decreased intracellular heme $b$ concentration, expressed as per mole carbon. Chlorophyll a (chl a) to heme $b$ ratios in E. huxleyi and $D$. tertiolecta increased in response to limited light and nutrient availability, but slightly decreased or did not change in the diatoms and the cyanophyte Synechococcus sp. WH7803. The heme b:particulate organic carbon (POC) and chl $a$ :heme $b$ ratios in the North Atlantic were within the range observed in phytoplankton cultures. In the surface mixed layer, decreases in heme $b:$ POC ratios were linked to decreases in nutrient concentrations. Chl a:heme $b$ ratios increased with depth and were thus primarily affected by light availability. Relative relationships between heme $b$, chl $a$ and POC in the North Atlantic likely represented a change in the ability of cells to undertake cellular processes driven by chl a (light harvesting) and heme $b$ (e.g. electron transport) according to ambient light and nutrient conditions.

KEY WORDS: Hemoprotein · Iron · Electron transport · Cytochrome · Nutrient limitation · Celtic Sea

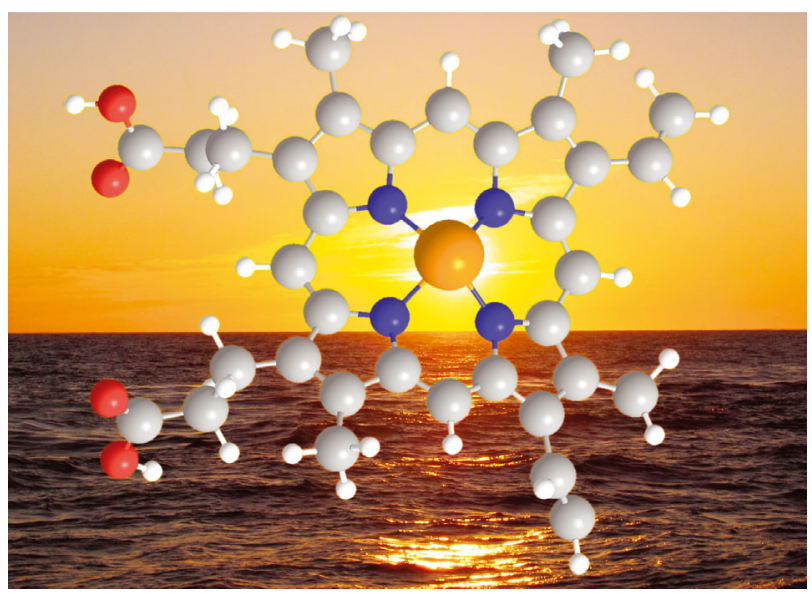

In heme $b$, the central iron atom (orange) is bound to four nitrogen atoms (blue) of a tetrapyrrole ring composed of carbon (grey), hydrogen (white) and oxygen (red) atoms.

Image: M. Gledhill and T. Bibby

\section{INTRODUCTION}

Iron is among Earth's most abundant elements; however, iron concentrations can be extremely low $\left(<0.2 \mathrm{nmol} \mathrm{l}^{-1}\right)$ in oceanic surface waters (De Baar \& De Jong 2001). Iron is involved in many fundamental biological processes, including photosynthesis, respiration, nitrogen fixation and nitrate reduction (Geider \& LaRoche 1994, Sunda 2012). Hemoproteins form an important reservoir of iron in oceanic microorganisms and are 1 of 3 types of iron-containing proteins, along with iron sulphur and iron oxygen proteins (Frausto da Silva \& Williams 2001). Hemoproteins are a functionally diverse group involved 
in electron transfer, substrate oxidation and oxygen transport, storage and reduction (Chapman et al. 1997). In particular, hemoproteins facilitate the 2 most fundamental processes in biology: respiratory and photosynthetic electron transfer via $b$ - and $c$ type cytochromes (Mochizuki et al. 2010). However, knowledge of the abundance of hemoproteins in phytoplankton and their distribution in the marine environment is currently very limited (Gledhill 2007, Saito et al. 2011), although it has been shown that low iron concentrations result in reduced abundance of other iron proteins in phytoplankton (Bibby et al. 2009, Marchetti et al. 2009, Saito et al. 2011, Richier et al. 2012).

Hemoproteins contain heme(s) (iron-porphyrin complexes) as the prosthetic group. Hemes act as a direct source of iron for some marine heterotrophic bacteria (Hopkinson et al. 2008), and heme uptake genes have been identified in diverse regions of the ocean (Desai et al. 2012, Hopkinson \& Barbeau 2012). Hemes are highly toxic to cells when not associated with proteins, meaning heme abundance is tightly linked to hemoprotein abundance (Espinas et al. 2012). The many functions attributed to hemoproteins arise from variations in iron ligation and charge state, as well as the addition of different substituents on the tetrapyrrole ring (Frausto da Silva \& Williams 2001). Four specific heme structures are relatively common in organisms (hemes $a, b, c$ and $d$ ) of which heme $b$ (iron protoporphyrin IX) is the most widespread (Espinas et al. 2012). Heme $b$ is associated with glo- bins, catalases, peroxidases, cytochrome P450 and $b$-type cytochromes (Mochizuki et al. 2010). Hemes are closely related to the photosynthetic pigments chlorophyll and phycobilin, which are produced via the same tetrapyrrole biosynthetic pathway as heme (Cornah et al. 2003, Mochizuki et al. 2010). During tetrapyrrole biosynthesis, iron or magnesium is chelated into protoporphyrin IX to produce heme or chlorophyll, respectively. A complex feedback mechanism determines the allocation of protoporphyrin IX to either the heme or chlorophyll branch of the synthesis pathway (Vavilin \& Vermaas 2002).

The aim of our study was to investigate heme $b$ in phytoplankton and particulate material in the ocean. The abundance of heme $b$ and its response to changes in iron, nitrate and light conditions was explored in 6 species of phytoplankton originating from the North Atlantic. Species from different phylogenetic groups were chosen to allow comparison with previous published work on the abundance of heme $b$-containing proteins (e.g. Greene et al. 1991, 1992, Strzepek \& Harrison 2004, Suggett et al. 2007, Allen et al. 2008). Relationships between the concentration of heme $b$, chlorophyll $a$ ( $\mathrm{chl} a$ ), particulate organic carbon (POC) and particulate organic nitrogen (PON) were investigated. In addition, particulate concentrations of heme $b$ were determined in samples collected from the Celtic Sea, part of the northwest European Shelf Sea, and from a cross-sectional (sub-)tropical North Atlantic transect (NA transect) close to the 24th parallel (Fig. 1).

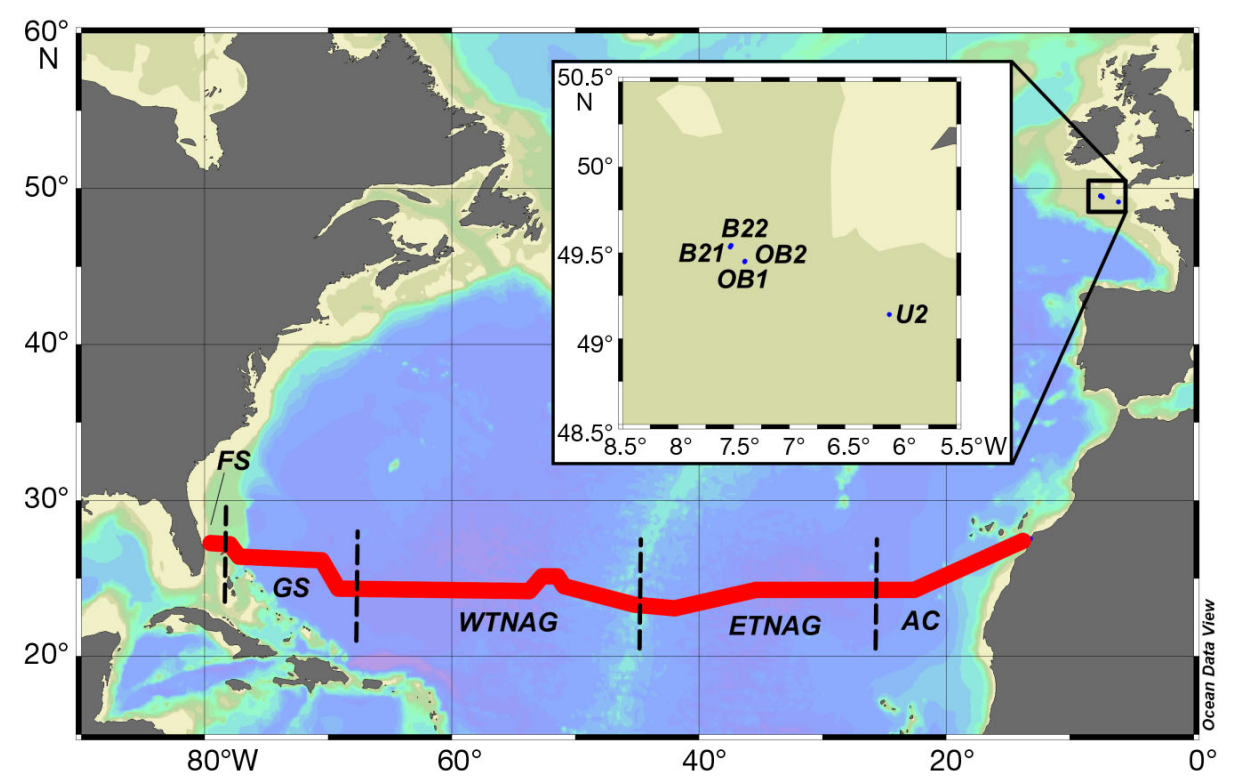

Fig. 1. Stations sampled in the Celtic Sea (inset) and the route of the North Atlantic transect (NA transect; red line). Five oceanographic regions of the NA transect were defined as the Florida Straits (FS), Gulf Stream (GS), West Tropical North Atlantic Gyre (WTNAG), East Tropical North Atlantic Gyre (ETNAG) and Azores Current (AC). Colours represent bathymetry (blue: > 2000 m; green: 250-2000 m; grey: <250 m) 


\section{MATERIALS AND METHODS}

\section{Phytoplankton growth conditions and sampling}

Batch cultures of Dunaliella tertiolecta Butcher (1959) (PCC83, CCAP 19/6B), Emiliania huxleyi B11 (CCAP 920/8), Thalassiosira weissflogii (Grunow in van Heurck) Fryxell \& Hasle (1977) (PCC541, CCAP 1085/1), T. oceanica (Hustedt) Hasle et Heimdal (PCC692, originally CCMP1005) and Phaeodactylum tricornutum Bohlin 1897 (PCC670, CCMP 632) were grown in an experimental medium prepared from filtered seawater $(0.2 \mu \mathrm{m}$, Sartobran, Sartorius) collected from the North Atlantic Subtropical Gyre (24.1-29.5 ${ }^{\circ} \mathrm{N}$ and 23.4-27.6 $6^{\circ} \mathrm{W}$, January 2005). Basal nutrient concentrations were $0.15 \pm 0.04 \mu \mathrm{mol} \mathrm{l} \mathrm{l}^{-1}$ nitrate, $0.06 \pm 0.06 \mu \mathrm{mol} \mathrm{l}^{-1}$ phosphate, $0.5 \pm 0.03 \mu \mathrm{mol}$ $\mathrm{l}^{-1}$ silicate and $0.54 \pm 0.05 \mathrm{nmol} \mathrm{l^{-1 }}$ dissolved iron (errors are expressed as SD, unless otherwise stated). All chemicals and consumables were purchased from Thermo Fisher Scientific unless otherwise stated. The culture medium was enriched with 150 (high) or 15 (low) $\mu \mathrm{mol} \mathrm{l} \mathrm{l}^{-1}$ nitrate, $10 \mu \mathrm{mol} \mathrm{l^{-1 }}$ phosphate, $100 \mu \mathrm{mol} \mathrm{l^{-1 }}$ silicate, $100 \mu \mathrm{mol} \mathrm{l^{-1 }}$ ethylenediamine tetraacetic acid (EDTA), $50 \mathrm{nmol}^{-1}$ cobalt, $100 \mathrm{nmol}$ $\mathrm{l}^{-1}$ molybdenum, $20 \mathrm{nmol} \mathrm{l}^{-1}$ copper, $115 \mathrm{nmol} \mathrm{l}^{-1}$ manganese, $80 \mathrm{nmol} \mathrm{l}^{-1}$ zinc, $10 \mathrm{nmol} \mathrm{l}^{-1}$ selenium and $\mathrm{f} / 2$ vitamins. Iron $(\mathrm{Fe})$ was added separately from a stock solution of $4.5 \mathrm{mmol} \mathrm{l}^{-1}$ FeEDTA at concentrations between $0.5 \mathrm{nmol} \mathrm{l}^{-1}$ and $1.5 \mu \mathrm{mol} \mathrm{l}^{-1}$ added iron. Trace metals were removed from the nutrient stock solutions using a cation chelating resin (chelex 100, Sigma). Artificial seawater (Wilson et al. 1996) was used to culture Synechococcus sp. WH7803 (CCMP1334). Iron was omitted from the initial media recipe and added separately from a stock FeEDTA solution $\left(1.2 \mathrm{mmol} \mathrm{l}^{-1}\right)$ to create 3 different iron concentrations between $12 \mathrm{nmol} \mathrm{l}^{-1}$ and $1.2 \mu \mathrm{mol} \mathrm{l}^{-1}$. Concentrations of iron not complexed by EDTA, expressed as $\mathrm{pFe}$ ' and equal to $-\log \left[\mathrm{Fe}^{\prime}\right]$, were calculated according to Sunda et al. (2005), taking into account the photochemical lability of FeEDTA complexes. Concentrations of $\mathrm{Fe}^{\prime}>0.7 \mathrm{nmol} \mathrm{l}^{-1}$ exceeded the solubility of iron in seawater media (Sunda et al. 2005), and thus include precipitated iron oxyhydroxides. Media were filtered $(0.2 \mu \mathrm{m}$, Minisart, Sartorius) into sterile polycarbonate culture vessels (EasyFlasks) and stored at $4^{\circ} \mathrm{C}$ prior to use.

Cultures were maintained at the iron concentrations used in the experiments for a minimum of 6 generations prior to the experimental run. For low nitrate and low light experiments, cultures were grown for a minimum of 3 generations prior to experimental runs. As cultures reached the end of the exponential phase, phytoplankton were transferred to fresh medium to obtain an initial biovolume close to $1.5 \times$ $10^{6}{\mu \mathrm{m}^{3}}^{3} \mathrm{l}^{-1}$. Dunaliella tertiolecta, Thalassiosira weissflogii, T. oceanica, Emiliania huxleyi and Phaeodactylum tricornutum were grown at $19^{\circ} \mathrm{C}$ under cool white fluorescent lights at $150 \mu \mathrm{mol}$ quanta $\mathrm{m}^{-2} \mathrm{~s}^{-1}$ (high light) or $45 \mu \mathrm{mol}$ quanta $\mathrm{m}^{-2} \mathrm{~s}^{-1}$ (low light) on a 12:12 h light:dark cycle. Synechococcus sp. WH7803 was grown at $22^{\circ} \mathrm{C}$ under $30 \mu \mathrm{mol}$ quanta $\mathrm{m}^{-2} \mathrm{~s}^{-1}$ light on a 12:12 h light:dark cycle (Wilson et al. 1996).

Cell density and biovolume were monitored daily on fresh culture samples with a Beckman Coulter counter (Multisizer 3, Meritics) using $3 \mathrm{~mol} \mathrm{l}^{-1} \mathrm{NaCl}$ solution as diluent and electrolyte. The maximum quantum yield of PSII $\left(F_{\mathrm{v}} / F_{\mathrm{m}}\right)$ of dark-adapted cells were determined in the mid-exponential phase using a FAST ${ }^{\text {tracka }}$ II Fast Repetition Rate fluorometer (Chelsea Technologies; Kolber et al. 1998, Ross et al. 2008). Cultures were filtered onto glass microfibre filters (MF300, nominal pore size $0.7 \mu \mathrm{m}$, Fisher Scientific) for the determination of heme $b$ (10 to $200 \mathrm{ml}$ ), the photosynthetic protein PsbA (D1 protein of PSII: 50 to $200 \mathrm{ml}$ ), chl a (10 to $30 \mathrm{ml}$ ) and POC and PON (hereafter POC/N: 10 to $200 \mathrm{ml}$ ) towards or at the end of the exponential phase. Filters for POC/N were ashed $\left(450^{\circ} \mathrm{C}, 16 \mathrm{~h}\right)$ prior to use. Filters for subsequent heme $b$ and PsbA analysis were stored at $-80^{\circ} \mathrm{C}$, whilst filters for chl $a$ and $\mathrm{POC} / \mathrm{N}$ were stored at $-20^{\circ} \mathrm{C}$. Cultures were filtered onto $25 \mathrm{~mm}, 0.7 \mu \mathrm{m}$ pore size polycarbonate membrane filters (Sterlitech) for the determination of particulate iron $\left(\mathrm{Fe}_{\mathrm{part}}\right)$. Filters for $\mathrm{Fe}_{\mathrm{part}}$ were soaked sequentially for $1 \mathrm{wk}$ in $6 \mathrm{~mol} \mathrm{l}^{-1}$ and $1 \mathrm{~mol}$ $\mathrm{l}^{-1}$ quartz distilled $\mathrm{HCl}$, then rinsed thoroughly with high purity water (MilliQ, Millipore) prior to use.

\section{Field sampling}

Samples were collected at 5 stations in the Celtic Sea between 27 July and 3 August 2005 and at 133 stations in the (sub-)tropical North Atlantic (NA transect) between 5 January and 15 February 2010 (Fig. 1). Six samples station ${ }^{-1}$ were obtained in the Celtic Sea, while up to 4 samples station ${ }^{-1}$ were collected along the NA transect. Seawater from depths $<200 \mathrm{~m}$ was obtained using 201 bottles (Ocean Test Equipment) mounted on a stainless steel CTD rosette. Particulate samples for the determination of heme $b$ were obtained by filtration of seawater onto glass microfibre filters (2 to 4 l). Sampling and analysis of chl $a$, 
POC/N and pigments in the Celtic Sea have been described previously (Hickman et al. 2009). Phytoplankton biomass (PhyC) was calculated from cell counts and cell volumes obtained by light microscopy (see Poulton et al. 2010) and flow cytometry (Hickman et al. 2009). Seawater samples for the determination of chl a $(200 \mathrm{ml})$ and POC/N (2 to 4 l) were filtered onto glass microfibre filters during the NA transect. Filters for POC/N analysis were previously ashed at $450^{\circ} \mathrm{C}$ for $12 \mathrm{~h}$. Filtered samples for heme $b$, chl $a$ and POC/N were stored at $-80^{\circ} \mathrm{C}$ prior to laboratory analysis. On the NA transect, surface mixed layer (SML) samples for nitrate+nitrite (hereafter referred to as nitrate) and phosphate were analysed using a $2 \mathrm{~m}$ liquid wave guide capillary cell set-up according to Patey et al. (2010). Detection limits were $2 \mathrm{nmol} \mathrm{l}^{-1}$ for nitrate and $1 \mathrm{nmol} \mathrm{l}^{-1}$ for phosphate, with a linear range up to $300 \mathrm{nmol}^{-1}$. Nitrate and phosphate in samples from below the SML were determined according to the methods of Sanders \& Jickells (2000).

\section{Determination of heme $b$}

Heme $b$ was analysed within 4 mo of sample collection. Heme $b$ was extracted into a $1 \mathrm{ml}$ ammoniacal $\left(20 \mathrm{mmol} \mathrm{l}^{-1}\right)$ detergent $(\mathrm{pH}=9.8$, EMPIGEN, $2.5 \% \mathrm{v}: \mathrm{v}$, Sigma) solution by ultrasonication (15 min) and centrifugation (12000 × $g, 10 \mathrm{~min})$. EMPIGEN (a zwitterionic detergent) was used in this study as it is more readily miscible in water than Triton X100 (Gledhill 2007). Comparison of heme $b$ concentrations determined using EMPIGEN and Triton X100 for Dunaliella tertiolecta showed that results were not significantly different (Student's $t$-test, $\mathrm{n}=4)$. Extracts were filtered $(0.2 \mu \mathrm{m}$, Minisart, Sartorius) prior to analysis. Heme $b$ in the extract was quantified using high performance liquid chromatography with diode array spectrophotometry (HPLC-DAD; Gledhill 2007). Heme $b$ extraction efficiencies were examined for $D$. tertiolecta and Phaeodactylum tricornutum grown using resource-replete conditions and for the hemoproteins catalase and horse radish peroxidase (HRP). For the phytoplankton, heme $b$ was extracted from filters using acid acetone (80:20 v:v acetone:1.6 mol $\left.1^{-1} \mathrm{HCl}\right)$ and ammoniacal detergent as described above. Catalase (10 $\left.\mathrm{mg} \mathrm{ml}^{-1}\right)$ and HRP (1.2 $\mathrm{mg} \mathrm{ml}^{-1}$ ) were dissolved in a $20 \mathrm{mmol} \mathrm{l}^{-1}$ TRIS- $\mathrm{HCl}$ solution ( $\mathrm{pH}$ 8.5). Twenty $\mu \mathrm{l}$ of solution were added to $980 \mu \mathrm{l}$ of acidified acetone or ammoniacal detergent. Solutions were ultrasonicated on ice for $15 \mathrm{~min}$. Ammo- niacal detergent extracts were analysed directly by HPLC-DAD. For acidified acetone solutions, $100 \mu \mathrm{l}$ of each extract were added to $900 \mu \mathrm{l}$ ammoniacal detergent prior to HPLC-DAD analysis. Samples were processed in triplicate.

\section{Determination of $\mathrm{chl}$ a, POC/N, PsbA and particulate iron}

Chl a was extracted in 90\% acetone and darkrefrigerated for $24 \mathrm{~h}$ (Welschmeyer 1994). A fluorometer (Turner Designs 10-AU) was used to determine chl a concentration, calibrated using pure chl a spinach standard (Sigma). A molar mass of $893.5 \mathrm{~g} \mathrm{~mol}^{-1}$ was used for conversion of chlorophyll in $\mu \mathrm{g} \mathrm{l}^{-1}$ to molar equivalents of chl a. Prior to analysis of POC/N samples, inorganic carbon was removed by acidification with sulphurous acid $\left(\mathrm{H}_{2} \mathrm{SO}_{3}\right)$ under vacuum for 24 to $48 \mathrm{~h}$ (Verardo et al. 1990). POC and PON were analysed via an elemental analyser (Thermo Finnegan Flash EA1112) using acetanilide as the calibration standard. Samples from cultures of Thalassiosira oceanica and Emiliania huxleyi at selected iron and light treatments (see below) were analysed in duplicate for the PSII protein PsbA (D1). PsbA was quantified by immunoblotting as described previously (Brown et al. 2008, Richier et al. 2012, Ryan-Keogh et al. 2012).

Particulate iron was determined by inductively coupled plasma-mass spectrometry (ICP-MS, X Series, Thermo Scientific). Only filters from cultures at $\mathrm{Fe}_{\mathrm{T}} \leq 45 \mathrm{nmol} \mathrm{l^{-1 }}$ were analysed in order to minimise the contribution of iron adsorbed onto the surface of the cell (Ho et al. 2003). Samples for Fe $\mathrm{p}_{\text {part }}$ from cultures of Thalassiosira weissflogii and Phaeodactylum tricornutum were not collected. Filters were placed in acid-washed $20 \mathrm{ml}$ PFA digestion vials with $2 \mathrm{ml}$ of concentrated redistilled $\mathrm{HNO}_{3}$ (Savillex, QMX) and heated to $80^{\circ} \mathrm{C}$ for $24 \mathrm{~h}$. The filter was removed from the vial and the $\mathrm{HNO}_{3}$ evaporated off $\left(80^{\circ} \mathrm{C}\right.$, overnight). The residue was dissolved in $10 \mathrm{ml}$ of $2 \%(\mathrm{v}: \mathrm{v}) \mathrm{HNO}_{3}$ and spiked with indium $\left(1 \mu \mathrm{g}^{-1}\right)$ as an internal standard prior to analysis. Concentrations were calculated from a calibration curve constructed from iron standards (ICP-MS, Inorganic Ventures) after correction for drift and matrix effects using the internal standard. Procedural filter blanks, prepared by filtering 50 to $100 \mathrm{ml}$ of culture media, were determined to be $109 \pm$ 28 pmol per filter $(n=10)$, resulting in a detection limit (3x standard deviation of blank) for iron of $8.5 \mathrm{nmol} \mathrm{l}^{-1}$. 


\section{Statistical analysis}

Statistical analysis was carried out using Sigma Plot ${ }^{\circledR}$ v.11. Errors are expressed as \pm SD, unless otherwise stated. Analysis of variance (ANOVA) was used to examine significant differences between growth rates and $F_{\mathrm{v}} / F_{\mathrm{m}}$ in phytoplankton experiments, with the high iron, high nitrate, high light treatment serving as the control condition (i.e. resource replete). For field data, Student's $t$-test was used to examine differences in parameters between the SML and the deep chlorophyll maximum (DCM). Where data sets were not normally distributed, rank sum tests were applied. The variations in parameters across the NA transect were examined using 1-way ANOVAs. Pearson Product Moment Correlations (r) were used to measure the strength of association between parameters. Contour plots were produced using Ocean Data View v.4.3.2 (http://odv.awi.de).

\section{RESULTS}

\section{Extraction efficiency of ammoniacal detergent solution}

Recoveries of hemin added to samples of Dunaliella tertiolecta and Phaeodactylum tricornutum were $103 \pm 15 \%(\mathrm{n}=7)$. Comparison of extraction efficiencies of heme $b$ from catalase, HRP, $D$. tertiolecta and $P$. tricornutum using ammoniacal detergent and acidified acetone, a commonly used alternative extraction protocol (Stillman \& Gassman 1978, Yu \& Weinstein 1997, Masuda \& Takahashi 2006, Espinas et al. 2012) are given in Table 1. Both techniques exhibited some variability in the amount of heme $b$ extracted from the soluble proteins, although acidified acetone was more efficient in all cases. The efficiency of extraction for catalase, D. ter-

Table 1. Dunaliella tertiolecta and Phaeodactylum tricornutum. Comparison of heme $b$ concentrations extracted from phytoplankton and heme $b$ proteins (catalase and horse radish peroxidase, HRP) using ammoniacal detergent, AD (2.5\% v:v EMPIGEN:20 mmol $\left.\mathrm{l}^{-1} \mathrm{NH}_{4} \mathrm{OH}\right)$ and acidified acetone, AA (80:20 v:v acetone: $1.6 \mathrm{~mol} \mathrm{l}^{-1} \mathrm{HCl}$ ). Data are means $\pm \mathrm{SD}$

\begin{tabular}{|llccc|}
\hline & \multicolumn{2}{c}{$\begin{array}{l}\text { Heme } b \text { in extract } \\
\left(\mathrm{nmol}^{-1}\right)\end{array}$} & AD:AA & $\begin{array}{c}\text { Expected heme } \\
b \text { concentration } \\
\left(\mathrm{nmol}^{-1}\right)\end{array}$ \\
& $\mathrm{AD}$ & $\mathrm{AA}$ & & \\
\hline $\begin{array}{l}\text { Dunaliella tertiolecta } \\
\begin{array}{l}\text { Phaeodactylum } \\
\text { tricornutum }\end{array}\end{array}$ & $65 \pm 39$ & $80 \pm 47$ & 0.81 & \\
$\begin{array}{l}\text { Catalase } \\
\text { HRP }\end{array}$ & $16 \pm 2$ & $107 \pm 2$ & 0.83 & 320 \\
\hline
\end{tabular}

tiolecta and P. tricornutum with ammoniacal detergent was $82 \pm 0.01 \%$ of that obtained with acidified acetone, while the extraction efficiency from HRP was less (Table 1).

\section{Heme $b$ in cultured phytoplankton}

Effects of varying iron concentration on heme $b$

All species examined exhibited decreased cell size (see Table S1 in the supplement at www.int-res. com/articles/m483p001_supp.pdf), growth rates $(\mu$, $\left.\mathrm{d}^{-1}\right)$ and maximum quantum yield of PSII $\left(F_{\mathrm{v}} / F_{\mathrm{m}}\right)$ at lower iron concentrations (Table 2). The exception was Thalassiosira weissflogii, which in fact could not be sustained for greater than 3 transfers at $5 \mathrm{nmol}^{-1}$ $\mathrm{Fe}_{\mathrm{T}}$ and only showed a reduction in cell numbers and cell size at $45 \mathrm{nmol} \mathrm{l^{-1 }} \mathrm{Fe}_{\mathrm{T}}$ (Table S1). $F_{\mathrm{v}} / F_{\mathrm{m}}$ is not presented for Synechococcus sp. WH7803, as saturation of the PSII reaction centre was incomplete. Of the species grown in this study, the coccolithophore Emiliania huxleyi and the pennate diatom Phaeodactylum tricornutum tolerated the lowest iron concentrations, with sustained, albeit reduced, growth at $0.5 \mathrm{nmol} \mathrm{l}^{-1} \mathrm{Fe}_{\mathrm{T}}$ (Table 2). This trend is similar to previous observations (Brand et al. 1983, Sunda \& Huntsman 1995, Bowler et al. 2010). Thus, results for growth and $F_{\mathrm{v}} / F_{\mathrm{m}}$ at low iron concentrations were consistent with iron-limited phytoplankton cultures.

Cellular chl $a$ (Fig. 2A) and heme $b$ (Fig. 2B) concentrations, expressed as $\mu \mathrm{mol} \mathrm{mol}{ }^{-1} \mathrm{C}$, varied 4-fold between species, decreasing with iron concentration except for Thalassiosira weissflogii (Table 2). Under high iron and light conditions, chl $a$ :heme $b$ ratios (Fig. 2C) ranged from $84 \pm 20$ for Dunaliella tertiolecta to $29 \pm$ 11 for Synechococcus sp. WH7803. Emiliania huxleyi and $D$. tertiolecta showed increased chl a:heme $b$ ratios in response to decreased iron concentration, with a maximum ratio of $243 \pm 28$ for $E$. huxleyi, a 3-fold increase compared to iron-replete conditions. In contrast, chl a:heme $b$ ratios of the diatoms and Synechococcus sp. WH7803 decreased at low iron concentrations.

Particulate $\mathrm{Fe}: \mathrm{C}$ ratios ranged from $3.5 \pm 0.1$ for Synechococcus to 20-22 $\mathrm{mmol} \mathrm{mol}^{-1}$ for Dunaliella tertiolecta, Emiliania huxleyi and Thalassiosira oceanica. These ratios were within the ranges observed previously for phytoplankton cultures grown at low iron concentrations 
Table 2. Maximum growth rate $\left(\mu_{\max }\right)$, maximum quantum yield $\left(F_{\mathrm{v}} / F_{\mathrm{m}}\right)$, carbon to nitrogen ratio $(\mathrm{C}: \mathrm{N})$, chl a concentration per unit carbon (chl $a: C)$ and nitrogen (chl $a: N)$ and heme $b$ concentration per unit carbon (heme $b: C$ ) and nitrogen (heme $b: N$ ) determined in the late exponential phase for Dunaliella tertiolecta, Emiliania huxleyi, Thalassiosira weissflogii, T. oceanica, Phaeodactylum tricornutum and Synechococcus sp. WH7803 cultures grown under varying iron concentrations and high light conditions. pFe : $-\log \left[\right.$ inorganic Fe]; Fe ${ }_{\mathrm{T}}$ : total dissolved iron. Data are mean $\pm \mathrm{SD}$. ${ }^{*}$ Significant differences in growth rate and $F_{\mathrm{v}} / F_{\mathrm{m}}$ values compared to the highest iron cultures (p $<0.01$ )

\begin{tabular}{|c|c|c|c|c|c|c|c|c|}
\hline $\begin{array}{l}\text { Species / treatment } \\
\left(\text { in nmol l-1 } \mathrm{Fe}_{\mathrm{T}}\right)\end{array}$ & $\mathrm{pFe}$ & $\begin{array}{l}\text { Growth rate } \\
\left(\mu_{\max } d^{-1}\right)\end{array}$ & $F_{\mathrm{v}} / F_{\mathrm{m}}$ & $\begin{array}{c}\mathrm{C}: \mathrm{N} \\
\left(\mathrm{mol} \mathrm{mol}{ }^{-1}\right)\end{array}$ & $\begin{array}{c}\text { Chl a:C } \\
\left(\mu \mathrm{mol} \mathrm{mol}^{-1}\right)\end{array}$ & $\begin{array}{c}\text { Chl a:N } \\
\left(\mu \mathrm{mol} \mathrm{mol}^{-1}\right)\end{array}$ & $\begin{array}{c}\text { Heme } b: C \\
\left(\mu \mathrm{mol} \mathrm{mol}{ }^{-1}\right)\end{array}$ & $\begin{array}{c}\text { Heme } b: \mathrm{N} \\
\left(\mu \mathrm{mol} \mathrm{mol}{ }^{-1}\right)\end{array}$ \\
\hline \multicolumn{9}{|l|}{ Dunaliella tertiolecta } \\
\hline 5 & 11.7 & $0.61 \pm 0.11^{*}$ & $0.37 \pm 0.02^{*}$ & $5.72 \pm 0.33$ & $336 \pm 35.3$ & $1912 \pm 111$ & $1.81 \pm 0.31$ & $10.3 \pm 1.69$ \\
\hline 45 & 10.8 & $1.06 \pm 0.05$ & $0.52 \pm 0.01^{*}$ & $5.94 \pm 0.04$ & $426 \pm 5.42$ & $2535 \pm 30.0$ & $4.94 \pm 0.92$ & $29.4 \pm 5.67$ \\
\hline 1500 & 9.2 & $0.94 \pm 0.03$ & $0.55 \pm 0.01$ & $6.15 \pm 0.05$ & $460 \pm 22.1$ & $2826 \pm 157$ & $5.60 \pm 1.02$ & $34.4 \pm 6.26$ \\
\hline \multicolumn{9}{|l|}{ Emiliania huxleyi } \\
\hline 0.5 & 12.7 & $0.67 \pm 0.05^{*}$ & $0.34 \pm 0.02^{*}$ & $7.19 \pm 0.26$ & $95.2 \pm 8.47$ & $683 \pm 36.5$ & $0.38 \pm 0.07$ & $2.67 \pm 0.64$ \\
\hline 5 & 11.7 & $0.99 \pm 0.04^{*}$ & $0.36 \pm 0.01$ & $7.87 \pm 0.23$ & $128 \pm 4.24$ & $1007 \pm 3.49$ & $0.91 \pm 0.04$ & $7.00 \pm 0.21$ \\
\hline 45 & 10.8 & $1.35 \pm 0.03^{*}$ & $0.40 \pm 0.02$ & $7.21 \pm 0.01$ & $214 \pm 0.25$ & $1544 \pm 0.05$ & $2.56 \pm 0.37$ & $17.7 \pm 3.30$ \\
\hline 1500 & 9.2 & $1.26 \pm 0.05$ & $0.39 \pm 0.02$ & $7.49 \pm 0.05$ & $218 \pm 1.99$ & $1631 \pm 3.79$ & $2.70 \pm 0.23$ & $20.0 \pm 2.30$ \\
\hline \multicolumn{9}{|c|}{ Thalassiosira weissflogii } \\
\hline 45 & 10.8 & $0.97 \pm 0.07$ & $0.46 \pm 0.02^{*}$ & $9.29 \pm 0.40$ & $222 \pm 20.3$ & $2063 \pm 269$ & $3.29 \pm 1.24$ & $30.9 \pm 12.5$ \\
\hline 188 & 10.1 & $1.06 \pm 0.14$ & $0.50 \pm 0.01$ & $8.80 \pm 0.22$ & $204 \pm 44.8$ & $1788 \pm 357$ & $4.59 \pm 0.29$ & $40.4 \pm 3.16$ \\
\hline 1500 & 9.2 & $1.23 \pm 0.14$ & $0.49 \pm 0.01$ & $8.44 \pm 0.07$ & $180 \pm 17.5$ & $1519 \pm 149$ & $2.82 \pm 0.18$ & $23.8 \pm 1.38$ \\
\hline \multicolumn{9}{|c|}{ Thalassiosira oceanica } \\
\hline 5 & 11.7 & $0.36 \pm 0.07^{*}$ & $0.38 \pm 0.02^{*}$ & $6.44 \pm 0.32$ & $60.5 \pm 8.90$ & $388 \pm 37.9$ & $2.51 \pm 0.71$ & $16.9 \pm 4.61$ \\
\hline 45 & 10.8 & $0.75 \pm 0.06$ & $0.48 \pm 0.01^{*}$ & $6.61 \pm 0.21$ & $173 \pm 4.15$ & $1142 \pm 63.7$ & $3.43 \pm 0.22$ & $21.7 \pm 0.81$ \\
\hline 188 & 10.1 & $0.91 \pm 0.05$ & $0.49 \pm 0.01$ & $7.01 \pm 0.07$ & $202 \pm 10.6$ & $1419 \pm 88.7$ & $3.65 \pm 1.02$ & $25.4 \pm 9.09$ \\
\hline 1500 & 9.2 & $0.84 \pm 0.02$ & $0.50 \pm 0.01$ & $7.38 \pm 0.07$ & $175 \pm 3.71$ & $1293 \pm 15.8$ & $4.53 \pm 0.34$ & $34.1 \pm 4.08$ \\
\hline \multicolumn{9}{|c|}{ Phaeodactylum tricornutum } \\
\hline 0.5 & 12.7 & $0.63 \pm 0.11^{*}$ & $0.31 \pm 0.02^{*}$ & $6.22 \pm 0.38$ & $22.0 \pm 1.68$ & $137 \pm 6.24$ & $0.99 \pm 0.25$ & $6.19 \pm 1.70$ \\
\hline 5 & 11.7 & $1.03 \pm 0.03$ & $0.45 \pm 0.02^{*}$ & $7.31 \pm 0.22$ & $19.5 \pm 1.24$ & $142 \pm 9.24$ & $1.45 \pm 0.42$ & $10.8 \pm 3.41$ \\
\hline 45 & 10.8 & $1.04 \pm 0.04$ & $0.48 \pm 0.01^{*}$ & $8.64 \pm 0.48$ & $92.5 \pm 6.90$ & $603 \pm 371$ & $2.65 \pm 0.60$ & $22.8 \pm 4.55$ \\
\hline 1500 & 9.2 & $0.92 \pm 0.09$ & $0.49 \pm 0.02$ & $8.26 \pm 0.33$ & $123 \pm 11.2$ & $1018 \pm 130$ & $3.42 \pm 1.06$ & $28.4 \pm 9.48$ \\
\hline \multicolumn{9}{|c|}{ Synechococcus sp. WH7803 } \\
\hline 12 & 10.0 & $0.49 \pm 0.04^{*}$ & & $5.36 \pm 2.23$ & $12.4 \pm 4.55$ & $37.9 \pm 13.7$ & $0.72 \pm 0.30$ & $2.17 \pm 0.86$ \\
\hline 120 & 9.0 & $0.83 \pm 0.06$ & & $6.43 \pm 0.54$ & $44.9 \pm 15.0$ & $215 \pm 87.3$ & $2.10 \pm 0.78$ & $10.2 \pm 4.56$ \\
\hline 1200 & 8.0 & $0.89 \pm 0.09$ & & $6.55 \pm 0.48$ & $56.9 \pm 17.7$ & $279 \pm 145$ & $1.94 \pm 0.86$ & $9.34 \pm 4.89$ \\
\hline
\end{tabular}

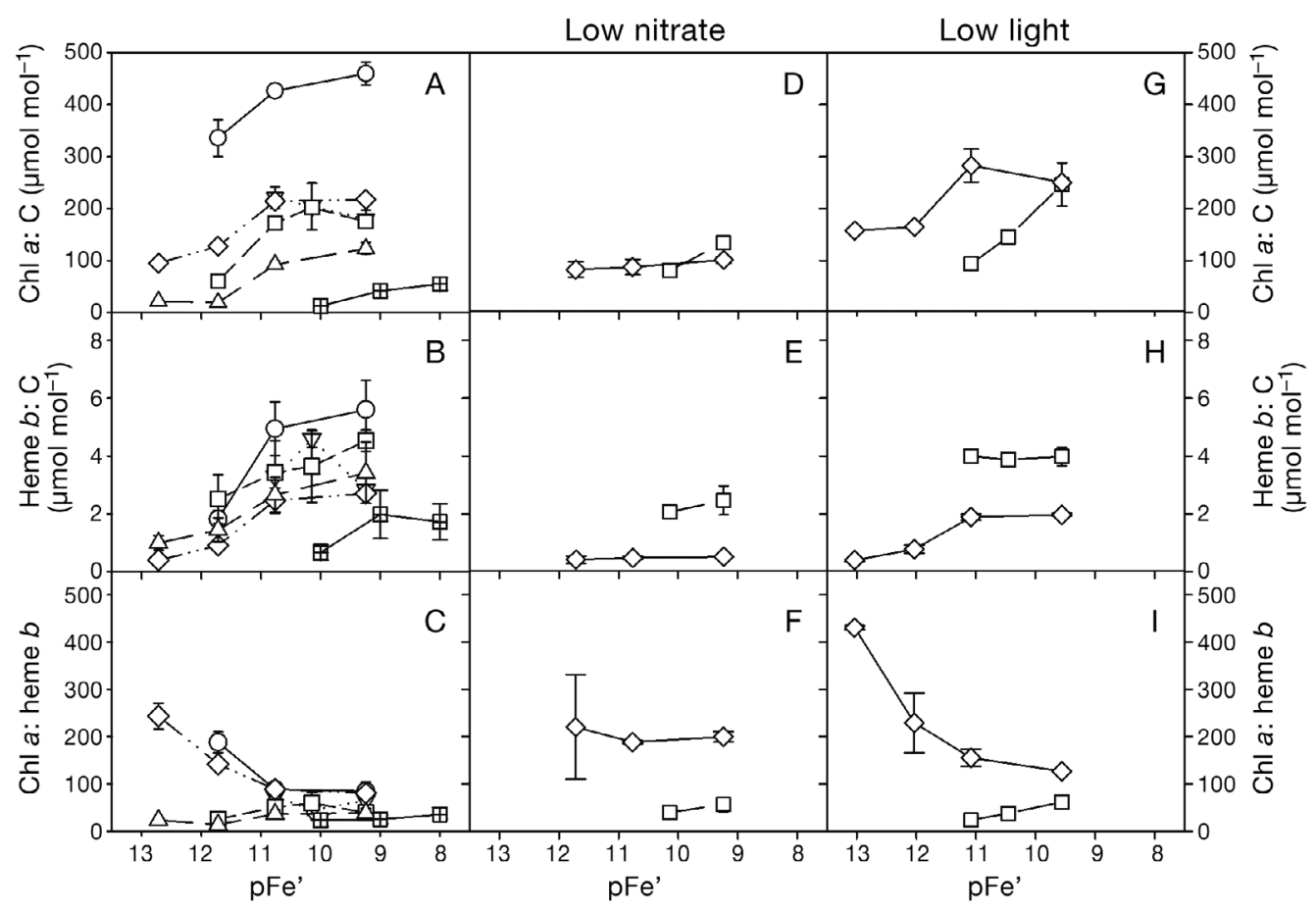

Fig. 2. Ratios of chl $a: C$, heme $b: C$ and chl a:heme $b$ for Dunaliella tertiolecta (open circles), Emiliania huxleyi (diamonds), Thalassiosira weissflogii (inverted triangles), $T$. oceanica (squares), Phaeodactylum tricornutum (triangles) and Synechococcus sp. WH7803 (crossed squares) grown under varying iron concentrations (A-C). Eukaryotes were grown with $150 \mu \mathrm{mol}$ quanta $\mathrm{m}^{-2} \mathrm{~s}^{-1}$, while Synechococcus was grown with $30 \mu \mathrm{mol}$ quanta $\mathrm{m}^{-2}$ $\mathrm{s}^{-1}$. Further experiments were conducted on E. huxleyi and T. oceanica grown under $(\mathrm{D}-\mathrm{F})$ low nitrate $\left(15 \mu \mathrm{mol} \mathrm{^{-1 }}\right)$ and with $(\mathrm{G}-\mathrm{I})$ low light $\left(45 \mu \mathrm{mol}\right.$ quanta $\mathrm{m}^{-2} \mathrm{~s}^{-1}$ ). $\mathrm{pFe}^{\prime}$ represents - $\log [$ inorganic $\mathrm{Fe}]$. Error bars: $\pm 1 \mathrm{SD}$ 
Table 3. Particulate iron:carbon $\left(\mathrm{Fe}_{\mathrm{part}}: \mathrm{C}, \mu \mathrm{mol} \mathrm{mol}{ }^{-1}\right)$ ratios and the contribution (\%) of heme $b$ to particulate iron in phytoplankton cultures. Results are presented only for cultures where the total dissolved iron $\left(\mathrm{Fe}_{\mathrm{T}}\right)$ concentration was $\leq 45 \mathrm{nmol} \mathrm{l}^{-1}$, to minimise any contribution from iron adsorbed on the surface of the cell. Samples were not collected for Phaeodactylum tricornutum and Thalassiosira weissflogii. Samples where $\mathrm{Fe}_{\text {part }}$ was below the detection limit are indicated by $<$ dl. Data are means $\pm \mathrm{SD}$

\begin{tabular}{|c|c|c|}
\hline $\begin{array}{l}\text { Species / treatment } \\
\left(\text { in nmol } 1^{-1} \mathrm{Fe}_{\mathrm{T}}\right)\end{array}$ & $\begin{array}{c}\mathrm{Fe}_{\text {part }}: \mathrm{C} \\
\left(\mu \mathrm{mol} \mathrm{mol}^{-1}\right)\end{array}$ & $\begin{array}{c}\text { Heme } b: F e_{\text {part }} \\
(\%)\end{array}$ \\
\hline \multicolumn{3}{|l|}{ Dunaliella tertiolecta } \\
\hline 5 & $<\mathrm{dl}$ & \\
\hline 45 & $21 \pm 12$ & $26 \pm 8$ \\
\hline \multicolumn{3}{|l|}{ Emiliania huxleyi } \\
\hline 0.5 & $7 \pm 3$ & $6 \pm 2$ \\
\hline 5 & $4 \pm 2$ & $27 \pm 11$ \\
\hline 45 & $20 \pm 12$ & $14 \pm 6$ \\
\hline \multicolumn{3}{|c|}{ Emiliania huxleyi (low nitrate) } \\
\hline 5 & $<\mathrm{dl}$ & \\
\hline 45 & $50 \pm 27$ & $1.1 \pm 0.5$ \\
\hline \multicolumn{3}{|c|}{ Emiliania huxleyi (low light) } \\
\hline 0.5 & $<\mathrm{dl}$ & \\
\hline 5 & $7 \pm 5$ & $25 \pm 20$ \\
\hline 45 & $5 \pm 1$ & $40 \pm 5$ \\
\hline \multicolumn{3}{|c|}{ Thalassiosira oceanica } \\
\hline 5 & $21 \pm 11$ & $13 \pm 3$ \\
\hline 45 & $22 \pm 1$ & $16 \pm 1$ \\
\hline \multicolumn{3}{|c|}{$\begin{array}{l}\text { Thalassiosira oceanica } \\
\text { (low light) }\end{array}$} \\
\hline 45 & $<\mathrm{dl}$ & \\
\hline \multicolumn{3}{|c|}{ Synechococcus sp. WH7803 } \\
\hline 12 & $3.5 \pm 0.1$ & $10 \pm 2$ \\
\hline
\end{tabular}

(Table 3; Sunda \& Huntsman 1995, Ho et al. 2003, Twining et al. 2004). However, direct comparison of species (e.g. T. oceanica; Sunda \& Huntsman 1995) show differences that could be attributable to either methodological or experimental considerations (Hopkinson et al. 2012, Twining \& Baines 2013). Iron in heme $b$ was calculated to contribute between $6 \pm 2$ and $27 \pm 11 \%$ of the $\mathrm{Fe}_{\text {part }}$ determined in our experiments.

The ratio of chl a:PsbA in iron-replete conditions (1500 nmol $\mathrm{l}^{-1} \mathrm{Fe}_{\mathrm{T}}$ ) averaged $260 \pm 30$ for Emiliania huxleyi and $270 \pm 70$ for Thalassiosira oceanica (Table 4). For E. huxleyi, these ratios were lower than chl a:PSII ratios observed previously in the same strain (<488; Suggett et al. 2007), possibly as a result of lower cellular chl a content observed in this study (e.g. chl a cell ${ }^{-1}$ at $1500 \mathrm{nmol} \mathrm{l}^{-1} \mathrm{Fe}_{\mathrm{T}}, 45 \mu \mathrm{mol}$ quanta $\mathrm{m}^{-2} \mathrm{~s}^{-1}=67 \pm 1 \mathrm{fg}$ cell ${ }^{-1}$ ). For $T$. oceanica, chl a:PsbA ratios agreed well with previously reported values (213 to 273; Strzepek \& Harrison 2004). Heme $b$ was within an order of magnitude of PsbA concentrations (Table 4), and the ratio of heme $b$ :PsbA did not vary greatly with iron concentration. However, T. oceanica was observed to have a higher heme $b$ :PsbA ratio compared to E. huxleyi, averaging $7 \pm 3(\mathrm{n}=8)$ and $3.4 \pm 1.2(\mathrm{n}=6)$, respectively.

\section{Effect of low nitrate concentrations on heme $b$}

Emiliania huxleyi and Thalassiosira oceanica were selected for further experimentation at different nitrate and light levels, as these 2 species exhibited contrasting behaviours with respect to chl a:heme $b$ ratio (Fig. 2C). Experiments with low nitrate were undertaken at all previously described iron concentrations; however, here we only present data for cultures exhibiting increased carbon:nitrogen (C:N) ratios (Table 5, Fig. 2D-F), which were taken to be indicative of nitrate deficiency. As nitrate concentrations were suboptimal with respect to other nutrients, but not kept at minimal levels, these experiments represent a situation (such as a coastal environment) where nitrate is exhausted before other nutrients, although the results may not be representative of steady state nitrate-limited conditions. Growth rates for E. huxleyi were reduced compared with cultures grown at high nitrate, with a possible additional effect of in- 
Table 5. Emiliania huxleyi and Thalassiosira oceanica. Maximum growth rate $\left(\mu_{\max }\right)$, maximum quantum yield $\left(F_{\mathrm{v}} / F_{\mathrm{m}}\right)$, carbon to nitrogen ratio (C:N), chl a concentration per unit carbon ( $\mathrm{chl} a: \mathrm{C}$ ) and nitrogen (chl $a: \mathrm{N})$ and heme $b$ concentration per unit carbon (heme $b: \mathrm{C})$ and nitrogen (heme $b: C$ ) determined in the late exponential phase for cultures grown under varying iron concentrations and low light or low nitrate conditions. pFe represents $-\log [\text { inorganic } \mathrm{Fe}]_{;} \mathrm{Fe}_{\mathrm{T}}$ : total dissolved iron. Data are mean \pm SD. ${ }^{*}$ Significant differences in growth rates and $F_{\mathrm{v}} / F_{\mathrm{m}}$ compared to high nitrate, high light and high iron conditions $(\mathrm{p}<0.01)$

\begin{tabular}{|c|c|c|c|c|c|c|c|c|}
\hline $\begin{array}{l}\text { Species / treatment } \\
\left(\text { in nmol } 1^{-1} \mathrm{Fe}_{\mathrm{T}}\right)\end{array}$ & $\mathrm{pFe}$ & $\begin{array}{l}\text { Growth rate } \\
\left(\mu_{\max } d^{-1}\right)\end{array}$ & $F_{\mathrm{v}} / F_{\mathrm{m}}$ & $\begin{array}{c}\mathrm{C}: \mathrm{N} \\
\left(\mathrm{mol} \mathrm{mol} \mathrm{mol}^{-1}\right)\end{array}$ & $\begin{array}{c}\text { Chl a:C } \\
\left.(\mu \mathrm{mol} \mathrm{mol})^{-1}\right)\end{array}$ & $\begin{array}{c}\text { Chl a:N } \\
\left.(\mu \mathrm{mol} \mathrm{mol})^{-1}\right)\end{array}$ & $\begin{array}{c}\text { Heme b:C } \\
\left(\mu \mathrm{mol} \mathrm{mol}{ }^{-1}\right)\end{array}$ & $\begin{array}{l}\text { Heme } b: \mathrm{N} \\
\left(\mu \mathrm{mol} \mathrm{mol}{ }^{-1}\right)\end{array}$ \\
\hline \multicolumn{9}{|c|}{ Emiliania huxleyi (low nitrate) } \\
\hline 5 & 11.7 & $0.77 \pm 0.09^{*}$ & $0.39 \pm 0.02$ & $17.0 \pm 0.35$ & $83.0 \pm 14.8$ & $1407 \pm 222$ & $0.41 \pm 0.12$ & $7.02 \pm 2.52$ \\
\hline 45 & 10.8 & $0.92 \pm 0.04^{*}$ & $0.45 \pm 0.01^{*}$ & $17.0 \pm 2.79$ & $87.8 \pm 14.9$ & $1471 \pm 7.66$ & $0.48 \pm 0.06$ & $7.82 \pm 0.09$ \\
\hline 1500 & 9.2 & $0.88 \pm 0.08^{*}$ & $0.44 \pm 0.01$ & $16.0 \pm 0.25$ & $101 \pm 5.95$ & $1618 \pm 69.7$ & $0.51 \pm 0.01$ & $8.11 \pm 0.09$ \\
\hline \multicolumn{9}{|c|}{ Emiliania huxleyi (low light) } \\
\hline 0.5 & 13.0 & $0.49 \pm 0.06^{*}$ & $0.34 \pm 0.01^{*}$ & $6.48 \pm 0.05$ & $158 \pm 5.21$ & $1022 \pm 26.4$ & $0.39 \pm 0.04$ & $2.37 \pm 0.04$ \\
\hline 5 & 12.0 & $0.84 \pm 0.09^{*}$ & $0.37 \pm 0.01$ & $7.87 \pm 0.03$ & $165 \pm 4.80$ & $1298 \pm 33.1$ & $0.73 \pm 0.13$ & $5.86 \pm 1.74$ \\
\hline 45 & 11.1 & $1.15 \pm 0.05^{*}$ & $0.41 \pm 0.02$ & $7.72 \pm 0.15$ & $283 \pm 31.3$ & $2181 \pm 199$ & $1.90 \pm 0.12$ & $14.1 \pm 0.34$ \\
\hline 1500 & 9.6 & $1.12 \pm 0.01^{*}$ & $0.41 \pm 0.05$ & 8.10 & 250 & 2028 & $1.97 \pm 0.05$ & 15.7 \\
\hline \multicolumn{9}{|c|}{ Thalassiosira oceanica (low nitrate) } \\
\hline 188 & 10.1 & $0.88 \pm 0.09$ & $0.34 \pm 0.02^{*}$ & $21.4 \pm 0.04$ & $81.2 \pm 6.55$ & $1734 \pm 136$ & $2.06 \pm 0.11$ & $44.1 \pm 2.25$ \\
\hline 1500 & 9.2 & $0.89 \pm 0.12$ & $0.39 \pm 0.01$ & $12.8 \pm 0.78$ & $134 \pm 10.4$ & $1706 \pm 51.4$ & $2.47 \pm 0.50$ & $31.6 \pm 7.14$ \\
\hline \multicolumn{9}{|c|}{ Thalassiosira oceanica (low light) } \\
\hline 45 & 11.1 & $0.86 \pm 0.21$ & $0.45 \pm 0.01$ & $5.14 \pm 0.09$ & $93.6 \pm 8.95$ & $481 \pm 38.1$ & $3.99 \pm 0.20$ & $20.5 \pm 0.69$ \\
\hline 188 & 10.5 & $0.85 \pm 0.24$ & $0.49 \pm 0.01$ & $7.18 \pm 0.13$ & $146 \pm 11.3$ & $1047 \pm 100$ & $3.88 \pm 0.20$ & $27.9 \pm 1.92$ \\
\hline 1500 & 9.6 & $0.83 \pm 0.01$ & $0.49 \pm 0.01$ & $6.60 \pm 0.08$ & $246 \pm 41.3$ & $1625 \pm 291$ & $3.98 \pm 0.31$ & $26.3 \pm 2.38$ \\
\hline
\end{tabular}

creased growth with increased $\mathrm{Fe}_{\mathrm{T}}$ observable in these experiments. For T. oceanica, growth rates did not change at low nitrate (Table 5). Low nitrate resulted in an apparent increase in $F_{\mathrm{v}} / F_{\mathrm{m}}$ for E. huxleyi, but a decrease in $F_{\mathrm{v}} / F_{\mathrm{m}}$ for $T$. oceanica (Table 5).

Reduced nitrate concentrations led to a decrease in chl $a$ and heme $b$ concentrations per unit $C$ for both Emiliania huxleyi and Thalassiosira oceanica (Fig. 2D,E). Growth with low nitrate resulted in a decreased heme $b: \mathrm{N}$ ratio in $E$. huxleyi, while heme $b$ was conserved relative to the total organic nitrogen pool in T. oceanica (Tables $2 \& 5$ ). Chl $a$ to heme $b$ ratios increased at low nitrate concentrations for $E$. huxleyi, but no change was observed in T. oceanica cultures (Fig. 2F). Particulate iron concentrations were only obtained for E. huxleyi at $45 \mathrm{nmol} \mathrm{l}^{-1} \mathrm{Fe}_{\mathrm{T}}$, as $\mathrm{Fe}_{\text {part }}$ was below the detection limit at $5 \mathrm{nmol}^{-1}$ $\mathrm{Fe}_{\mathrm{T}}$. For E. huxleyi at $45 \mathrm{nmol} \mathrm{l^{-1 }} \mathrm{Fe}_{\mathrm{T}}$, the $\mathrm{Fe}_{\mathrm{part}}: \mathrm{C}$ ratio was higher than observed under nitrate-replete conditions, and the resultant heme $b$ :Fe $\mathrm{e}_{\text {part }}$ ratio was considerably lower (Table 3).

\section{Effect of low light levels on heme $b$}

Low light levels resulted in a significant reduction in growth for Emiliania huxleyi but, similar to reduced nitrate conditions, did not affect the growth of Thalassiosira oceanica (Table 5). Changes in $F_{\mathrm{v}} / F_{\mathrm{m}}$ were similar to those observed at high light levels between the different iron concentrations (Table 5). At low light, chl a concentration per unit $\mathrm{C}$ (chl a:C) increased by approximately 1.3 times for $E$. huxleyi at all iron concentrations, while chl a: $\mathrm{C}$ in $T$. oceanica only increased in the highest iron treatment (1500 nmol l-1 $\mathrm{Fe}_{\mathrm{T}}$, Fig. 2G). Decreasing light levels resulted in lower heme $b$ : $C$ ratios for $E$. huxleyi grown with high iron (1500 nmol $\left.\mathrm{l}^{-1} \mathrm{Fe}_{\mathrm{T}}\right)$, but had little effect on the heme $b$ concentration when grown under low iron and low light conditions (Fig. $2 \mathrm{H}$ ). The heme $b$ :C ratio for $T$. oceanica was similar under both low and high light conditions. For E. huxleyi, further increases in chl $a$ :heme $b$ ratio were observed under low light when compared to high light conditions (Fig. 2I). Decreasing light had no further effect on chl a:heme $b$ ratios for T. oceanica (Fig. 2I). For E. huxleyi grown at low light, $\mathrm{Fe}_{\text {part }}: \mathrm{C}$ and heme $b: \mathrm{Fe}_{\text {part }}$ ratios were similar to those observed at high light and $5 \mathrm{nmol} \mathrm{l}^{-1}$ $\mathrm{Fe}_{\mathrm{T}}$, but $\mathrm{Fe}_{\mathrm{part}}: \mathrm{C}$ decreased and heme $b: \mathrm{Fe}_{\text {part }}$ increased at $45 \mathrm{nmol} \mathrm{l}^{-1} \mathrm{Fe}_{\mathrm{T}}$ (Table 3). Concentrations of $\mathrm{Fe}_{\text {part }}$ in E. huxleyi grown with $0.5 \mathrm{nmol} \mathrm{l}^{-1} \mathrm{Fe}_{\mathrm{T}}$ and in T. oceanica cultures grown with low light were below the detection limit. Chl a:PsbA and heme $b$ :PsbA ratios at low light were similar to high light conditions, with the exception of $E$. huxleyi grown with $5 \mathrm{nmol} \mathrm{l}^{-1}$ $\mathrm{Fe}_{\mathrm{T}}$, where lower values were observed (Table 4).

\section{Heme $b$ concentrations in the North Atlantic Ocean}

Depth profiles for heme $b$, chl $a$ and chl $a$ :heme $b$ ratio for 5 shelf stations in the Celtic Sea and for an 


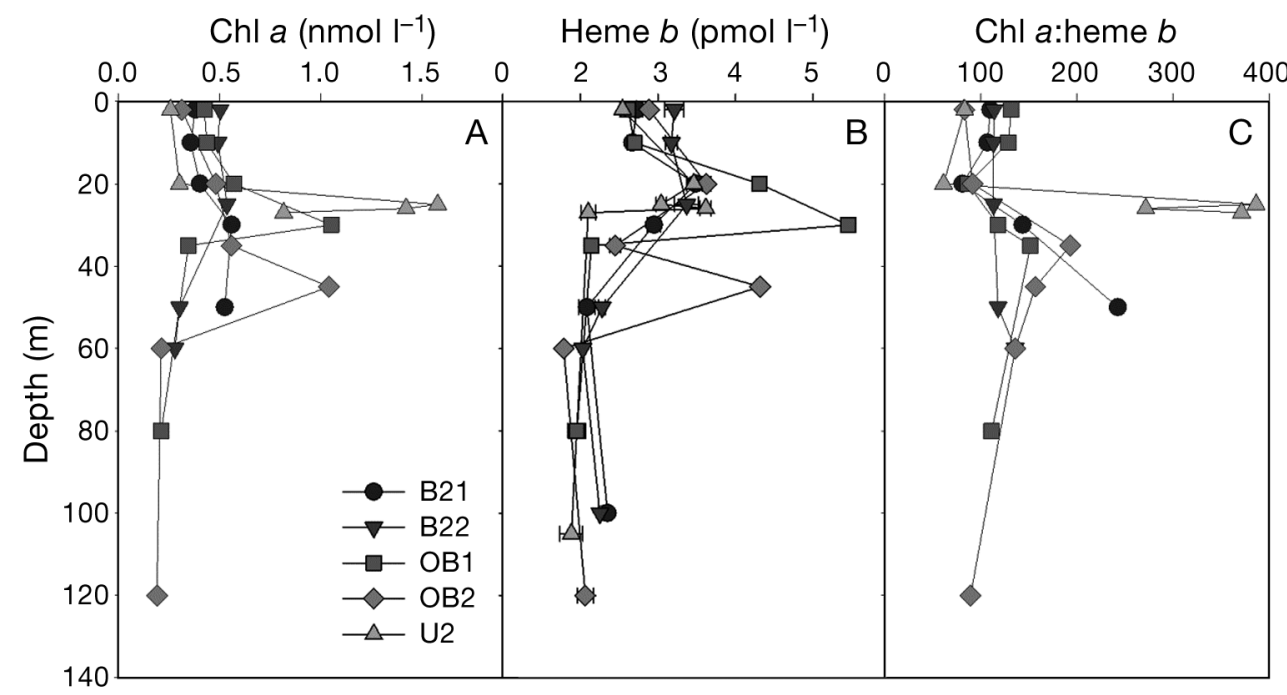

Fig. 3. Depth profiles of (A) chl a concentration, (B) heme $b$ and (C) chl $a$ :heme $b$ ratio for 5 stations in the Celtic Sea. Error bars for heme $b$ : range of duplicate measurements

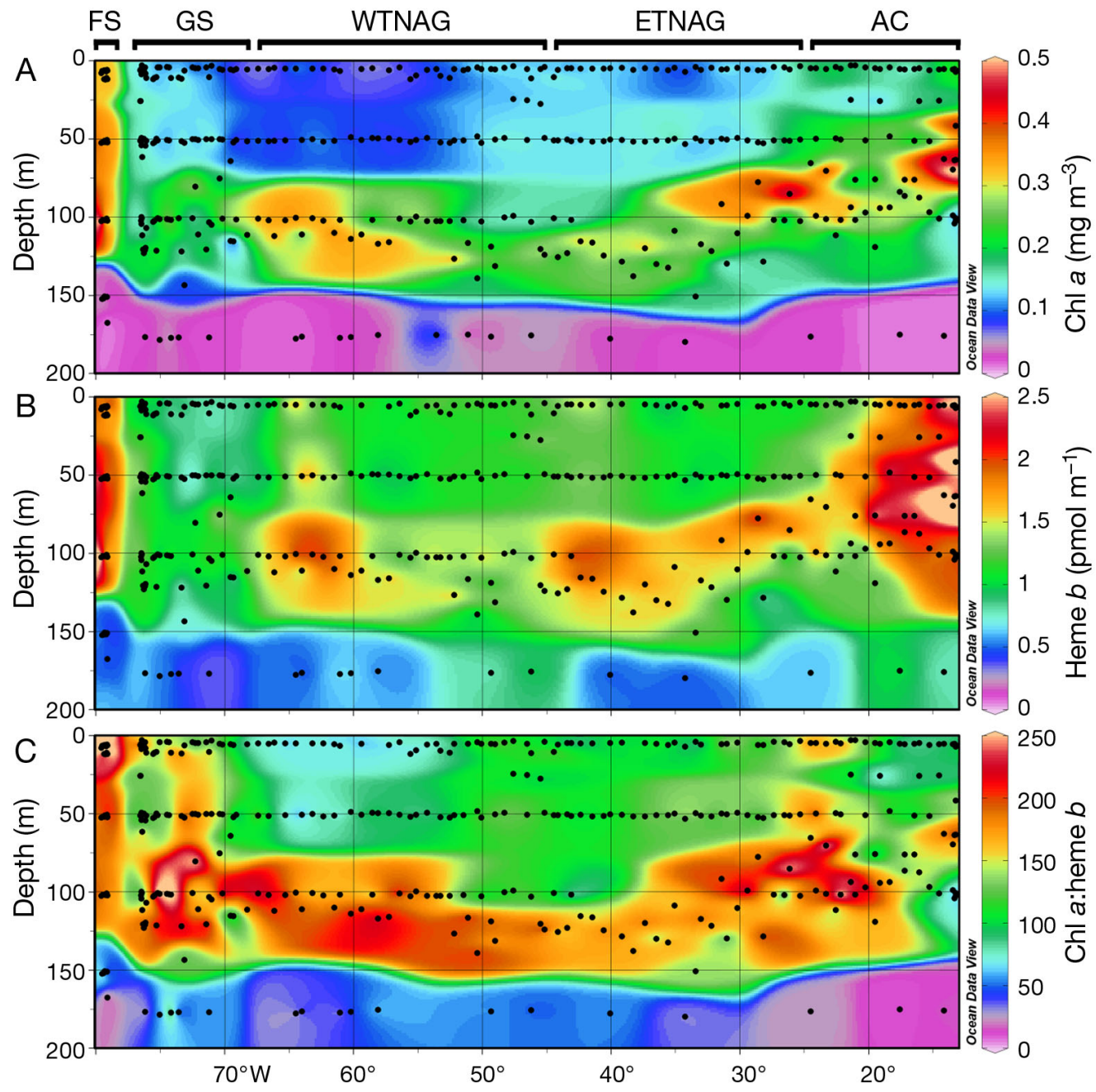

Fig. 4. Cross-sectional profiles $(<200 \mathrm{~m})$ from the North Atlantic transect (see Fig. 1) for (A) chl $a$ concentration, (B) heme $b$ concentration and $(\mathrm{C}) \mathrm{chl}$ a:heme $b$ ratio. Dots: sample positions. Abbreviations as in Fig. 1 
open-ocean NA transect are presented in Figs. $3 \& 4$ and in Tables S3 \& S4 in the Supplement (www. int-res.com/articles/suppl/m483p001_supp.pdf). Maximum heme $b$ concentrations coincided with maximum chl a concentrations, and there was an overall correlation between the 2 parameters $\left(\mathrm{r}^{2}=0.55, \mathrm{n}=\right.$ $376, \mathrm{p}<0.01)$. Nevertheless, chl a:heme $b$ ratios varied between 44 and 756, indicating important differences in the distributions of the 2 tetrapyrroles. In both the Celtic Sea and in the oligotrophic gyre on the NA transect, a DCM was observed below the SML. The phytoplankton community in the DCM was likely physiologically adapted to the ambient light and nutrient conditions (Agustí \& Duarte 1999, Robinson et al. 2006, Hickman et al. 2009); therefore, results obtained in the DCM are compared with those in the SML in order to assess any light-driven changes in heme $b$ in relation to POC and chl $a$.

\section{Celtic Sea}

The mean chl a concentration in samples from the Celtic Sea was $0.54 \pm 0.35 \mathrm{nmol} \mathrm{l}^{-1}\left(0.48 \pm 0.31 \mu \mathrm{g} \mathrm{l}^{-1}\right)$, with the highest concentration (1.6 nmol $\mathrm{l}^{-1}$ ) observed in the DCM (Fig. 3A). Heme $b$ averaged $3.7 \pm$ $1.7 \mathrm{pmol} \mathrm{l}^{-1}(\mathrm{n}=30)$ and, similar to $\mathrm{chl} a$, increased in the DCM (Fig. 3B) reaching a maximum concentration of $8.9 \mathrm{pmol} \mathrm{l}^{-1}$. POC, PON and pigment concentrations were determined in surface waters and the DCM (see Table S3 in the Supplement. Ratios of POC:PON averaged $7.1 \pm 0.7$ and decreased with increasing depth at all stations, although there was no significant difference between the SML and DCM. Ratios of heme $b$ :POC averaged $0.42 \pm 0.13 \mu \mathrm{mol}$ $\mathrm{mol}^{-1}$, while chl a:POC averaged $61 \pm 42 \mathrm{mmol}$ $\mathrm{mol}^{-1}(\mathrm{n}=16)$. There was a significant increase in chl a:POC in the DCM $\left(87 \pm 21 \mathrm{mmol} \mathrm{mol}^{-1}, \mathrm{n}=5\right)$ when compared with the SML $\left(38 \pm 6 \mathrm{mmol} \mathrm{mol}^{-1}\right.$, $\mathrm{n}=6$, rank sum test, $\mathrm{p}<0.01$ ) for all stations. Heme $b$ :POC ratios also increased at each station in the DCM (results not shown). Nevertheless, overall heme $b$ :POC ratios for all stations in the Celtic Sea were not significantly higher in the DCM $(0.54 \pm$ $\left.0.24 \mu \mathrm{mol} \mathrm{mol}{ }^{-1}, \mathrm{n}=5\right)$ than the SML $(0.4 \pm 0.1 \mu \mathrm{mol}$ $\left.\mathrm{mol}^{-1}, \mathrm{n}=6\right)$. The chl a:heme $b$ ratio varied with depth (Fig. 3C), with significantly higher ratios observed in the DCM $(203 \pm 102, \mathrm{n}=11)$ compared to the SML $(101 \pm 21, \mathrm{n}=12$, rank sum test, $\mathrm{p}<0.01)$. Increased chl a:heme $b$ ratios were reflected by increases in the ratio of other photosynthetic pigments (PSPs; peridinan, fucoxanthin, 19-butanoyloxyfucoxanthin, 19-hexanoyloxyfucoxanthin and chl $c_{2}$, $c_{3}$ and $b$ ) to heme $b$, which were also significantly higher in the DCM $(212 \pm 87, \mathrm{n}=6)$ compared to the SML $(100 \pm 25, \mathrm{n}=6$, Student's $t$-test, $\mathrm{p}<0.05)$. No significant trends were identified between the SML and DCM for non-photosynthetic caratenoids (alloxanthin, diadinoxanthin, prasinoxanthin, violaxanthin, zeaxanthin) compared to heme $b$. Estimates of phytoplankton biomass in the SML indicated that heme $b$ :PhyC at stations B21, B22, OB2 and U2 were approximately $0.28,0.65,0.45$ and $0.56 \mu \mathrm{mol} \mathrm{mol}^{-1}$, respectively, and were similar to those observed for heme $b: P O C$ in the region.

\section{(Sub-)tropical North Atlantic (NA transect)}

The NA transect, surveyed during January and February 2010, incorporated the Gulf Stream on the western margin, the oligotrophic (sub-)tropical North Atlantic gyre and the upwelling zone towards the eastern boundary of the Atlantic Ocean. The crosssectional profile of heme $b$ was spatially similar to chl $a$ across the NA transect (Fig. 4A,B), with a significant correlation $\left(\mathrm{r}^{2}=0.44, \mathrm{n}=357, \mathrm{p}<0.01\right)$. Chl $a$ and heme $b$ concentrations increased with depth, although the increase was more pronounced for chl $a$, with significant increases in heme $b$ in the DCM only apparent within the gyre (rank sum test, p < 0.01). In contrast, POC and PON did not increase in the DCM in any region when compared with the SML. POC:PON ratios averaged $7.6 \pm 1.4(\mathrm{n}=222)$ in the SML and decreased in the DCM to $7.0 \pm 0.9(n=68)$. The average heme $b$ :POC and chl $a$ :POC ratios for the NA transect were $0.63 \pm 0.4 \mu \mathrm{mol} \mathrm{mol}{ }^{-1}$ and $85 \pm 71 \mathrm{mmol} \mathrm{mol}^{-1}$, respectively; similar to those observed in the Celtic Sea. The nitracline generally coincided with the position of the thermocline (Fig. 5A,B), with increased nitrate concentrations observed within the DCM (up to $0.8 \mu \mathrm{mol} \mathrm{l^{-1 }}$ ). An increase in phosphate concentration throughout the water column $\left(>20 \mathrm{nmol} \mathrm{l}^{-1}\right)$ was observed in the east of the region (Fig. 5C).

The cross-sectional transect was divided into 5 regions (Fig. 1) based on the salinity and temperature of the SML (Fig. S1 in the Supplement) defined as: the Florida Straits (FS), the Gulf Stream (GS), the Western Tropical North Atlantic Gyre (WTNAG), the Eastern Tropical North Atlantic Gyre (ETNAG) and the Azores Current (AC). Mean heme $b$, chl $a$, POC and PON concentrations observed for each region are given in Table 6. Mean concentrations of heme $b$, chl $a$ and POC were highest in FS, while PON concentrations were highest in $\mathrm{AC}$. The average concentrations for each region suggested subtle differences 

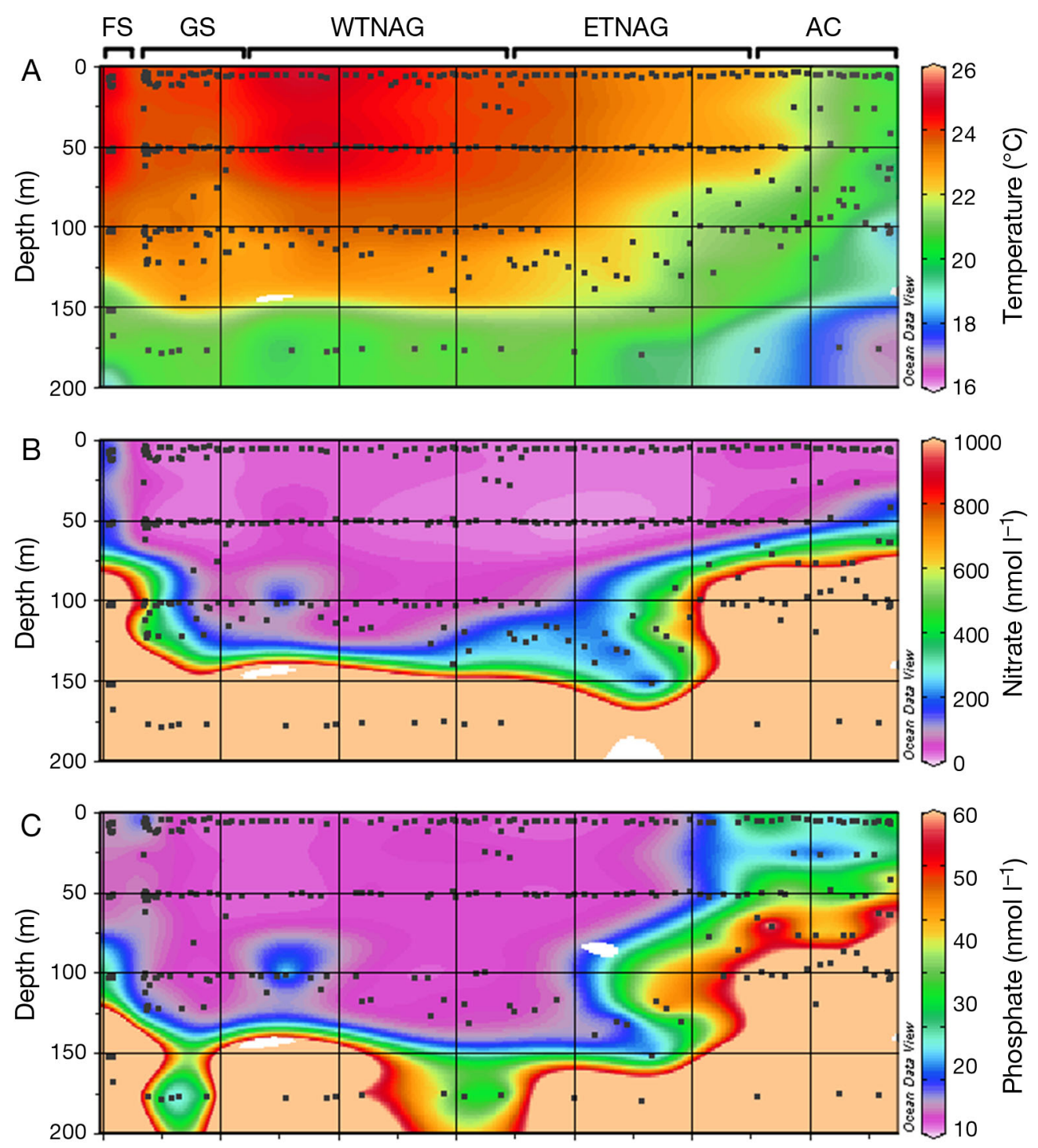

Fig. 5. Cross-sectional profiles $(<200 \mathrm{~m})$ from the North Atlantic transect (see Fig. 1) for (A) temperature, (B) nitrate concentration and (C) phosphate concentration. Dots: sample positions. Abbreviations as in Fig. 1

in relative distributions of heme $b$, chl $a$ and POC (Table 6). Very low chl a concentrations $(<0.1 \mathrm{nmol}$ $\mathrm{l}^{-1}$ ) were determined at $<50 \mathrm{~m}$ depth in the WTNAG (approximately $70-50^{\circ} \mathrm{W}$ ), leading to significantly lower chl a:POC ratios in the SML (Fig. 6A, ANOVA, $\mathrm{p}<0.01)$. In contrast, heme $b$ concentrations in the SML were lowest in GS (Fig. 6B, ANOVA, p < 0.01).

\section{DISCUSSION}

Our technique for heme $b$ analysis is based on separation by HPLC and detection by diode array spectrophotometry. HPLC combined with specific and sensitive detection offers advantages for the determination of coloured compounds, as it involves separation of heme $b$ from other pigments, which also absorb at similar wavelengths, coupled with positive identification of the heme $b$ peak from its retention time and absorption spectrum. Furthermore, there is potential for determination of other heme types (e.g. heme $a_{i}$ Lübben \& Morand 1994). However, acidified acetone, the extraction technique previously used to quantitatively extract heme $b$ from proteins and plant material (Stillman \& Gassman 1978, Yu \& Weinstein 1997, Masuda \& Takahashi 2006, Espinas et al. 2012), was not directly compatible with HPLC (Gledhill 2007). We found that chromatography of acidified 
Table 6. Mean concentrations for chl $a$, heme $b$, particulate organic carbon and nitrogen (POC and PON) and mean chl $a$ :heme $b$ ratios for the Celtic Sea (July to August 2005), Florida Straits, Gulf Stream, Eastern Tropical North Atlantic Gyre (ETNAG), Western Tropical North Atlantic Gyre (WTNAG) and the Azores Current of the North Atlantic transect (NA transect, January to February 2010; see Fig. 1) from the surface mixed layer (SML) and deep chlorophyll maximum (DCM). Data are mean \pm SD. Parentheses: no. of samples (n). Significant differences (rank sum test) between the SML and DCM are indicated $\left({ }^{* *} \mathrm{p}<0.01\right.$, $\left.{ }^{*} \mathrm{p}<0.05\right)$

\begin{tabular}{|c|c|c|c|c|c|}
\hline Region & $\begin{array}{c}\text { Chl a } \\
\left(\mathrm{nmol} \mathrm{l} \mathrm{l}^{-1}\right)\end{array}$ & $\begin{array}{l}\text { Heme } b \\
\left(\mathrm{pmol} \mathrm{l}^{-1}\right)\end{array}$ & $\begin{array}{l}\text { Chl a:heme } b \\
\left(\mathrm{~mol} \mathrm{~mol}^{-1}\right)\end{array}$ & $\begin{array}{c}\text { POC } \\
\left(\mu \mathrm{mol} \mathrm{l}^{-1}\right)\end{array}$ & $\begin{array}{c}\text { PON } \\
\left(\mu \mathrm{mol} \mathrm{l}^{-1}\right)\end{array}$ \\
\hline \multicolumn{6}{|l|}{ Celtic Sea } \\
\hline SML & $0.41 \pm 0.09(12)^{* *}$ & $4.1 \pm 0.8(12)$ & $101 \pm 21(12)^{* *}$ & $10.0 \pm 1.6(7)$ & $1.3 \pm 0.16(7)$ \\
\hline DCM & $0.9 \pm 0.4(10)$ & $4.7 \pm 2.2(10)$ & $203 \pm 102(11)$ & $10.6 \pm 2.9(5)$ & $1.6 \pm 0.6(5)$ \\
\hline \multicolumn{6}{|l|}{ Azores Current } \\
\hline SML & $0.23 \pm 0.09$ & $1.8 \pm 0.6(49)$ & $136 \pm 55.1$ & $2.8 \pm 0.9(45)$ & $0.39 \pm 0.13(46)$ \\
\hline $\mathrm{DCM}$ & $0.29 \pm 0.13$ & $2.0 \pm 0.8(14)$ & $155 \pm 73.0$ & $2.4 \pm 0.6(13)$ & $0.34 \pm 0.07(13)$ \\
\hline \multicolumn{6}{|l|}{ ETNAG } \\
\hline SML & $0.14 \pm 0.04(59)^{* * *}$ & $1.2 \pm 0.3(55)^{* *}$ & $116 \pm 30.2^{* *}$ & $2.1 \pm 0.3(49)^{*}$ & $0.31 \pm 0.06$ \\
\hline DCM & $0.29 \pm 0.08$ & $1.6 \pm 0.4(19)$ & $178 \pm 33.1$ & $1.9 \pm 0.4(14)$ & $0.31 \pm 0.09(14)$ \\
\hline \multicolumn{6}{|l|}{ WTNAG } \\
\hline SML & $0.10 \pm 0.03(70)^{* *}$ & $1.1 \pm 0.3(66)^{* *}$ & $198 \pm 36.9^{* *}$ & $2.2 \pm 0.5(60)^{* *}$ & $0.3 \pm 0.05(60)$ \\
\hline DCM & $0.30 \pm 0.07(28)$ & $1.5 \pm 0.4(28)$ & $92.8 \pm 27.1$ & $1.8 \pm 0.3(25)$ & $0.26 \pm 0.04$ \\
\hline \multicolumn{6}{|l|}{ Gulf Stream } \\
\hline SML & $0.13 \pm 0.03(74)^{* *}$ & $1.0 \pm 0.3(71)^{*}$ & $138 \pm 40.7(71)^{* *}$ & $2.1 \pm 0.7(68)^{* *}$ & $0.26 \pm 0.05$ \\
\hline DCM & $0.23 \pm 0.05$ & $1.1 \pm 0.2(16)$ & $220 \pm 95.6(16)$ & $1.8 \pm 0.5(17)$ & $0.24 \pm 0.07(17)$ \\
\hline \multicolumn{6}{|l|}{ Florida Straits } \\
\hline All & $0.33 \pm 0.13(31)$ & $1.9 \pm 1(31)$ & $226 \pm 159$ & $3.0 \pm 0.6(30)$ & $0.35 \pm 0.07(30)$ \\
\hline
\end{tabular}

acetone extracts requires dilution in order to reduce solvent content and ensure retention of heme on the column. However, dilution resulted in higher detection limits and thus limited application of the technique to open ocean samples. The more detailed examination of heme $b$ extraction efficiency undertaken here indicates that heme $b$ is not fully extracted from soluble proteins using ammoniacal detergent. However, in analogy to iron, the major portion of heme $b$ in phytoplankton is likely to be associated with photosynthetic membrane-bound proteins (Strzepek \& Harrison 2004). Detergents are commonly used to solubilise and sometimes deactivate membrane proteins (Seddon et al. 2004). Furthermore, heme $b$ has been observed to dissociate from cytochrome $b_{559}$, the heterodimer of the PsbE
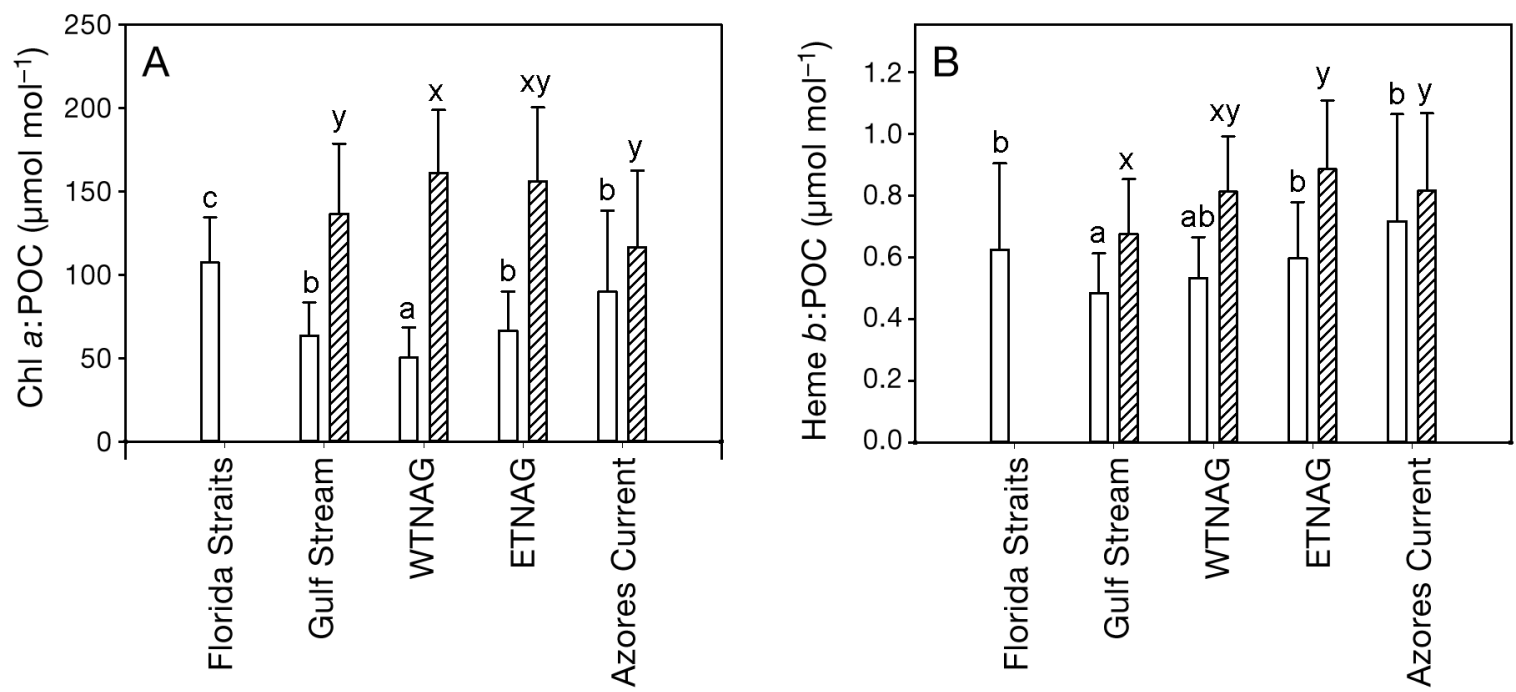

Fig. 6. Mean ratios of (A) chl a:particulate organic carbon (POC) and (B) heme $b$ :POC from 5 oceanographic regions on the North Atlantic transect (see Fig. 1). White bars: mean in the surface mixed layer (SML); hatched bars: mean within the deep chlorophyll maximum (DCM). Letters identify significantly different groups (1-way ANOVA applied separately to data from the SML $[\mathrm{a}-\mathrm{c}]$ and the DCM $[\mathrm{x}, \mathrm{y}], \mathrm{p}<0.05)$. Error bars: \pm 1 SD. WTNAG (ETNAG): West (East) Tropical North Atlantic Gyre 
and PsbF subunits of PSII, in non-ionic detergents at pH 10 (Weber et al. 2011). Clearly, heme $b$ proteins are differentially extracted in ammoniacal detergent, and it is possible that the different extraction efficiencies relate to protein solubility or the heme ligand environment within the protein (Weber et al. 2011). Results comparing heme $b$ with PsbA and Fe part (discussed below) suggest that overall heme $b$ may be extracted more efficiently from phytoplankton than from the soluble proteins examined in this study. However, incomplete extraction of heme $b$ with our technique means that the results obtained were operationally defined rather than fully quantitative, and further work is required in order to fully examine the factors controlling heme $b$ extraction.

\section{Heme $b$ in phytoplankton}

Knowledge of heme $b$ distributions in marine phytoplankton and the marine environment is extremely limited, with only 1 previously published preliminary study on phytoplankton cultures (Gledhill 2007). Heme $b$ concentrations in phytoplankton grown at the highest iron concentrations in our study were higher than previously reported values (Gledhill 2007). This may be related to the growth phase at which samples were obtained (late exponential phase compared with stationary phase) or improved preservation of samples prior to analysis $\left(-80^{\circ} \mathrm{C}\right.$ compared with $-20^{\circ} \mathrm{C}$ ). Heme $b$ : $\mathrm{C}$ ratios varied by an order of magnitude with both species and treatment, suggesting some variability in the intracellular abundance of this important compound (Fig. 2). Overall, heme $b$ made up between 1 and $40 \%$ (Table 3 ) of the particulate iron pool in the phytoplankton cultures, averaging $18 \pm 14 \%$. Although the particulate iron pool determined in this study may have included some surface adsorbed iron and heme $b$ extraction was not fully quantitative, the overall percentage of particulate iron from heme $b$ is very similar to theoretical heme $b: F e$ calculated for the electron transport chain (12 to 20\%; Gledhill 2007). Furthermore, the abundance of heme $b$ relative to PsbA for Thalassiosira oceanica and Emiliania huxleyi determined in this study (Table 4) appears reasonable. Inter-specific differences in heme $b$ :PsbA ratios were evident, possibly reflecting different photosynthetic strategies between phytoplankton species (Bowler et al. 2010). However, the ratios of heme $b$ :PsbA within species did not vary greatly in this study. Although a reduction in heme $b$ relative to PsbA was observed for $E$. huxleyi grown under low light and low iron condi- tions, the limited data available do not allow us to comment on the significance of these results.

With the exception of Thalassiosira weissflogii, the abundance of heme $b$ in the species examined in this study decreased when grown at lower iron concentrations (Fig. 2B). The diverse functionality of heme $b$-containing proteins (Mochizuki et al. 2010) would suggest that a decrease in intracellular heme $b$ concentration will compromise the organism's ability to undertake a range of fundamental processes (e.g. photosynthesis, respiration, scavenging reactive oxygen species, nitrate reduction). We observed a reduction in the maximum quantum yield of PSII $\left(F_{\mathrm{v}} / F_{\mathrm{m}}\right)$ in conjunction with a reduction in heme $b$ :C ratio in the eukaryotic phytoplankton (Fig. 7) when grown with different iron concentrations $\left(\mathrm{r}^{2}=0.76, \mathrm{n}=25\right.$, $\mathrm{p}<0.001$ ). Thus, under conditions of iron limitation, eukaryotic phytoplankton reduce their iron requirement by lowering cellular heme $b$ concentrations; this observation is correlated to a photosynthetic physiological response with implications for potential growth rates, possibly as a result of a reduction in photosystem cytochromes (Greene et al. 1991, Strzepek \& Harrison 2004, Falkowski \& Raven 2007). For growth at low nitrate concentrations, heme $b: \mathrm{C}$ and $F_{\mathrm{v}} / F_{\mathrm{m}}$ for $T$. oceanica fitted the linear trend observed for low light and low iron. In contrast, the results for Emiliania huxleyi indicated that $F_{\mathrm{v}} / F_{\mathrm{m}}$ and growth rate were not reduced to the same extent as for low iron treatments, despite similar heme $b$ :C ratios

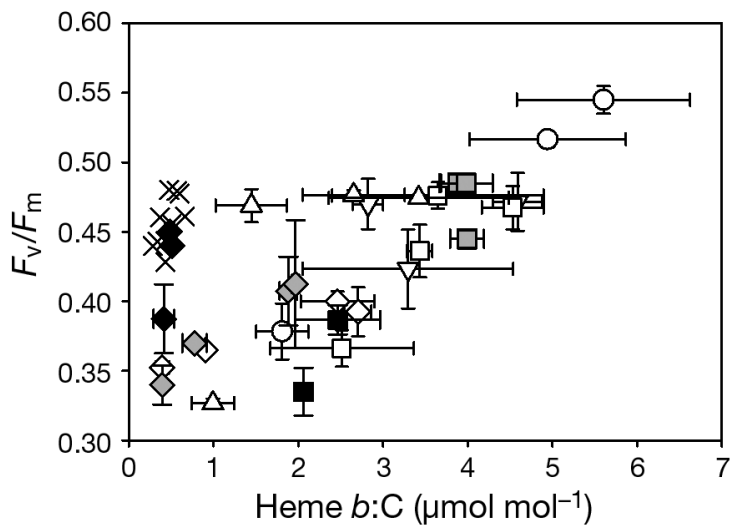

Fig. 7. Plot of heme $b$ :C against the maximum quantum yield of PSII $\left(F_{\mathrm{v}} / F_{\mathrm{m}}\right)$ for Dunialella tertiolecta (circles), Emiliania huxleyi (diamonds), Thalassiosira weissflogii (inverted triangles), T. oceanica (squares) and Phaeodactylum tricornutum (triangles) grown under varying iron concentrations. Open symbols: experiments undertaken at $150 \mu \mathrm{mol}$ quanta $\mathrm{m}^{-2}$ $\mathrm{s}^{-1}$ (high light); grey symbols: experiments undertaken at $45 \mu \mathrm{mol}$ quanta $\mathrm{m}^{-2} \mathrm{~s}^{-1}$ (low light); black symbols: experiments with $15 \mu \mathrm{mol} \mathrm{l}^{-1}$ nitrate (low nitrate) for E. huxleyi and T. oceanica. Crosses: values obtained from the Celtic Sea. Errors bars: $\pm 1 \mathrm{SD}$ 
(Table 5, Fig. 7). It is possible that E. huxleyi alter the distribution of heme $b$ and thus hemoproteins by, for example, reducing the abundance of eukaryotic nitrate reductase (cytochrome $b_{5}$ ), while maintaining photosynthetic cytochromes $b_{559}$ and $b_{6}$. Previous studies have indicated that the hemoprotein pool can be both tightly regulated and highly dynamic in marine phytoplankton (Greene et al. 1992, Saito et al. 2011); however, further analysis of specific heme proteins is necessary in order to confirm whether such tight controls on heme $b$ allocation exist in E. huxleyi.

Changes in the ratio of chl a to heme $b$ likely reflect changes in the ratio of heme $b$-containing proteins (e.g. cytb $_{6} f$, PSII, nitrate reductase) to those that are associated with chl a (PSI, PSII, light harvesting antenna). The ratios of PSI:cytb ${ }_{6}$ :PSII vary considerably between species, within species and with growth conditions (Raven 1990, Greene et al. 1991, Strzepek \& Harrison 2004, Suggett et al. 2007). In addition, light harvesting antenna complexes (LHCs), which can contain chl $a$, are induced or migrate when marine photosynthetic organisms are nutrient stressed (Bibby et al. 2001, 2009, Boekema et al. 2001, Eberhard et al. 2008). For the diatoms Thalassiosira weissflogii, T. oceanica and Phaeodactylum tricornutum and the cyanobacteria Synechococcus sp. WH7803, the decrease in heme $b$ when grown with low iron was matched or slightly exceeded by decreases in chl $a$, so that the chl $a$ :heme $b$ ratio was maintained or decreased. In contrast, the increased chl $a$ :heme $b$ ratios observed for low iron Emiliania huxleyi and Dunaliella tertiolecta cultures indicate further decreases in heme $b$ proteins relative to the chl $a$ containing proteins when iron stressed. The ability to maintain or increase the chl a:heme $b$ ratio did not appear to be linked to the ability of a species to tolerate low iron concentrations in culture. Instead, the diatoms (amongst the limited number of species examined to date) were found to be capable of consistently maintaining $\mathrm{chl}$ a:heme $b$ ratios, perhaps indicating a phylogenetic link. These species-specific observations in the regulation of chl $a$ and heme $b$ are likely a combination of both variation in ratios of heme $b$ to photosynthetic reaction centres (PSI and PSII) and, for the eukaryotes, to light-harvesting antenna systems, which contain differing amounts of chl $a$.

Our results for phytoplankton cultures indicate that the cellular abundance of heme $b$ varied with iron and nitrate availability for all species examined, and that the abundance of heme $b$ varied with respect to chl a and, by implication, the abundance of other photosynthetic proteins for Emiliania huxleyi and
Dunaliella tertiolecta. The physiological consequence of changes in both the cellular abundance of heme $b$ and chl $a$ :heme $b$ ratio will depend on how specific heme $b$ proteins are regulated. The major function of chl $a$ is harvesting light, while that of heme $b$ is electron transport, exchange and catalysis. The changing ratios of chl a:heme $b$ observed in $D$. tertiolecta and $E$. huxleyi thus represent changes in these fundamental cellular processes according to the available light and nutrient resources. However, further work directly determining the abundance of specific hemoproteins is necessary in order to fully understand the impact of reduced intracellular heme $b$.

\section{Heme $b$ in the North Atlantic Ocean}

The water column of the Celtic Sea becomes stratified in the summer, and the SML becomes deplete in macronutrients (Pingree et al. 1976). A DCM develops, which is strongly coupled to the thermocline (Hickman et al. 2009), and the phytoplankton community structure is determined by the availability of macronutrients and light (Hickman et al. 2009, Sharples et al. 2009). Therefore, the Celtic Sea is thought to be nitrate limited in the SML. Dissolved iron concentrations of $0.6 \pm 0.9 \mathrm{nmol} \mathrm{l}^{-1}$ have been reported for surface waters on the shelf (Ussher et al. 2007). Similarly, iron concentrations in the (sub-) tropical North Atlantic are typically >0.2 $\mathrm{nmol} \mathrm{l^{-1 }}$ (Wu et al. 2001, Sedwick et al. 2005, Measures et al. 2008, Rijkenberg et al. 2008, 2011), with exogenous iron supplied as a result of the sporadic deposition of Saharan dust to surface waters (Jickells et al. 2005, Rijkenberg et al. 2012). Both nitrate and phosphate concentrations are thought to limit productivity in the (sub-)tropical North Atlantic (Mills et al. 2004, Moore et al. 2006, Davey et al. 2008).

The mean heme $b$ concentration for the Celtic Sea was higher than that observed along the NA transect. Furthermore, heme $b$ concentration gradually decreased from East to West across the NA transect (excluding FS; Table 6). Heme $b$ concentrations are influenced by biomass, and, as hemoproteins are nearly ubiquitous in life, bacteria, phytoplankton and zooplankton contribute to the total heme $b$ pool. In this study, we filtered samples to focus on the $>0.7 \mu \mathrm{m}$ fraction, thereby (mostly) excluding bacteria. Heme $b$ was compared to chl $a$, which occurs only in phytoplankton, and POC/N, which includes detrital material in addition to zooplankton. Thus, the ratios of heme $b: \mathrm{POC} / \mathrm{N}$ and chl a:heme $b$ will not necessarily be similar to those observed in laboratory 
phytoplankton cultures. Nevertheless, the relative concentrations of heme $b$, chl $a$ and POC/N in the Celtic Sea and on the NA transect were within the range observed in our cultures. Comparison of heme $b$ :POC and $F_{\mathrm{v}} / F_{\mathrm{m}}$ showed that values were also similar to those observed for our nitrate-limited Emiliania huxleyi cultures (Fig. 7). Furthermore, a comparison of biomass with POC for the Celtic Sea showed good agreement (PhyC 98\% POC). This indicated that the major portion of POC was made up of phytoplankton biomass and that the influence of bacteria, detrital material and zooplankton on POC and heme $b$ concentrations determined in this region was minimal. The observed heme $b$ :POC and heme $b$ :PhyC ratios and the relationship between heme $b$ and $F_{\mathrm{v}} / F_{\mathrm{m}}$ in the Celtic Sea likely reflect nitratelimited phytoplankton, and are thus consistent with previous observations in the area (Hickman et al. 2009, 2012).

On the NA transect, the heme $b: \mathrm{POC}$ and chl $a$ : POC ratios were similar to those in the Celtic Sea, although the overall abundances of both heme $b$ and chl a were lower. Therefore, it is likely that, as for the Celtic Sea, heme $b$ was depleted with respect to biomass on the NA transect when compared to nutrientreplete phytoplankton. Furthermore, reductions in heme $b$ concentrations were observed towards the west of the transect (Table 6, Fig. 6), with lowest heme $b: \mathrm{POC}$ ratios in GS and a correlation between heme $b: \mathrm{POC}$ ratio and the macronutrients in the SML (nitrate: $\mathrm{r}=0.27, \mathrm{n}=205, \mathrm{p}<0.01$; phosphate: $\mathrm{r}=$ $0.373, \mathrm{n}=195, \mathrm{p}<0.01$ ).

While low nutrient concentrations resulted in decreased heme $b$ :POC in the SML of the North Atlantic, chl a:heme $b$ ratios were more strongly influenced by depth (Figs. 3C \& 4C). For the Celtic Sea, where pigment data are also available, the increase in chl a:heme $b$ ratio was reflected by an increase in the ratio of PSP:heme $b$. Higher chl $a$ : heme $b$ and PSP:heme $b$ ratios could be due to larger photosynthetic antenna size in the DCM and be indicative of a light-limited community (Hickman et al. 2009, 2012). However, heme b:POC ratios also showed a slight increase with depth, especially in the WTNAG and ETNAG of the NA transect (Table 6), indicating that increases in the cellular content of heme $b$ proteins such as photosynthetic cytochromes was also occurring within the DCM.

The chl a:heme $b$ ratios observed in the Celtic Sea and on the NA transect were similar and within the range observed in Emiliania huxleyi and Dunaliella tertiolecta laboratory cultures, but higher than the ratios observed for the diatoms or Synechococcus sp.
WH7803. Increased chl a:heme $b$ ratios in the Celtic Sea and NA transect likely reflected a mixed phytoplankton population which tightly regulated chl a and heme $b$ abundance. Furthermore, our results also indicate that allocation of resources to these tetrapyrroles and their associated proteins were likely driven by the ambient light and nutrient conditions.

\section{CONCLUSION}

In this study, we observed consistent trends in heme $b$ concentration in cultured phytoplankton and particulate material from the Atlantic Ocean. Heme $b$ relative to POC in phytoplankton and particulate material generally decreased in response to decreases in iron and nutrients, while a slight increase in heme $b$ in response to low light levels was observed at depth in the North Atlantic (sub-)tropical gyre. Changes observed in chl a:heme $b$ ratios appeared to be species specific and could be related to intracellular regulation of accessory LHCs and photosystem proteins. Our study has improved our knowledge of the response of heme $b$ in phytoplankton and particulate material to low nutrient and low light environments. However, as heme $b$ is known to facilitate the biological cycling of both carbon and nitrogen (e.g. Frausto da Silva \& Williams 2001) and may represent as much as $20 \%$ of the total cellular iron pool (this study), the relative abundance of this tetrapyrrole in the ocean also has important implications for the biogeochemical cycling of these elements, and consequently merits further study.

Acknowledgements. We thank the anonymous reviewers for their helpful comments on the manuscript. This work was supported by the Royal Society and Natural Environment Research Council (Grants NE/E013546/1 and NE/ F002971/1). We also thank the following people: D. Scanlan (University of Warwick) for his gift of Synechococcus sp. WH7803; the crew and scientists of the RRS 'Charles Darwin' (Celtic Sea) and RRS 'Discovery' (NA transect); S. Torres-Valdez for the micromolar nutrient data; A. Poulton for the microscopy and biomass data; B. Head for help with POC/N analysis; and J. Green for help with experiments on Thalassiosira weissflogii and Phaeodactylum tricornutum.

\section{LITERATURE CITED}

Agustí S, Duarte CM (1999) Phytoplankton chlorophyll a distribution and water column stability in the central Atlantic Ocean. Oceanol Acta 22:193-203

Allen AE, LaRoche J, Maheswari U, Lommer M and others (2008) Whole-cell response of the pennate diatom Phaeodactylum tricornutum to iron starvation. Proc Natl Acad Sci USA 105:10438-10443 
Bibby TS, Nield J, Barber J (2001) Iron deficiency induces the formation of an antenna ring around trimeric photosystem I in cyanobacteria. Nature 412:743-745

Bibby TS, Zhang Y, Chen M (2009) Biogeography of photosynthetic light-harvesting genes in marine phytoplankton. PLoS ONE 4:e4601

Boekema EJ, Hifney A, Yakushevska AE, Piotrowski M and others (2001) A giant chlorophyll-protein complex induced by iron deficiency in cyanobacteria. Nature 412: 745-748

Bowler C, Vardi A, Allen AE (2010) Oceanographic and biogeochemical insights from diatom genomes. Annu Rev Mar Sci 2:333-365

Brand LE, Sunda WG, Guillard RRL (1983) Limitation of marine phytoplankton reproductive rates by zinc, manganese, and iron. Limnol Oceanogr 28:1182-1198

Brown CM, MacKinnon JD, Cockshutt AM, Villareal TA, Campbell DA (2008) Flux capacities and acclimation costs in Trichodesmium from the Gulf of Mexico. Mar Biol 154:413-422

Chapman SK, Daff S, Munro AW (1997) Heme: the most versatile redox centre in biology? Met Sites Proteins Models 88:39-70

Cornah JE, Terry MJ, Smith AG (2003) Green or red: What stops the traffic in the tetrapyrrole pathway? Trends Plant Sci 8:224-230

- Davey M, Tarran GA, Mills MM, Ridame C, Geider RJ, LaRoche J (2008) Nutrient limitation of picophytoplankton photosynthesis and growth in the tropical North Atlantic. Limnol Oceanogr 53:1722-1733

De Baar HJW, De Jong JTM (2001) Distribution, sources and sinks of iron in seawater. In: Turner DR, Hunter KA (eds) The biogeochemistry of iron in seawater. Wiley, Chichester, p 123-253

Desai DK, Desai F, LaRoche J (2012) Factors influencing the diversity of iron uptake systems in aquatic microorganisms. Front Microbiol 3:362

Eberhard S, Finazzi G, Wollman FA (2008) The dynamics of photosynthesis. Annu Rev Genet 42:463-515

Espinas NA, Kobayashi K, Takahashi S, Mochizuki N, Masuda T (2012) Evaluation of unbound free heme in plant cells by differential acetone extraction. Plant Cell Physiol 53:1344-1354

Falkowski PG, Raven JA (2007) Aquatic photosynthesis. Princeton University Press, Princeton, NJ

Frausto da Silva JJR, Williams RJP (2001) The biological chemistry of the elements, 2nd edn. Oxford University Press, Oxford

Geider RJ, LaRoche J (1994) The role of iron in phytoplankton photosynthesis, and the potential for iron-limitation of primary productivity in the sea. Photosynth Res 39: 275-301

> Gledhill M (2007) The determination of heme $b$ in marine phyto- and bacterioplankton. Mar Chem 103:393-403

- Greene RM, Geider RJ, Falkowski PG (1991) Effect of iron limitation on photosynthesis in a marine diatom. Limnol Oceanogr 36:1772-1782

- Greene RM, Geider RJ, Kolber Z, Falkowski PG (1992) Ironinduced changes in light harvesting and photochemical energy-conversion processes in eukaryotic marine algae. Plant Physiol 100:565-575

Hickman AE, Holligan PM, Moore CM, Sharples J, Krivtsov V, Palmer MR (2009) Distribution and chromatic adaptation of phytoplankton within a shelf sea thermocline. Limnol Oceanogr 54:525-536
Hickman AE, Moore CM, Sharples J, Lucas MI, Tilstone GH, Krivtsov V, Holligan PM (2012) Primary production and nitrate uptake within the seasonal thermocline of a stratified shelf sea. Mar Ecol Prog Ser 463:39-57

> Ho TY, Quigg A, Finkel ZV, Milligan AJ, Wyman K, Falkowski PG, Morel FMM (2003) The elemental composition of some marine phytoplankton. J Phycol 39: 1145-1159

> Hopkinson BM, Barbeau KA (2011) Iron transporters in marine prokaryotic genomes and metagenomes. Environ Microbiol 14:114-128

> Hopkinson BM, Roe KL, Barbeau KA (2008) Heme uptake by Microscilla marina and evidence for heme uptake systems in the genomes of diverse marine bacteria. Appl Environ Microbiol 74:6263-6270

Hopkinson BM, Seegers B, Hatta M, Measures CI, Mitchell BG, Barbeau KA (2012) Planktonic C:Fe ratios and carrying capacity in the southern Drake Passage. Deep Sea Res II 90:102-111

> Jickells TD, An ZS, Andersen KK, Baker AR and others (2005) Global iron connections between desert dust, ocean biogeochemistry, and climate. Science 308:67-71

Kolber ZS, Prasil O, Falkowski PG (1998) Measurements of variable chlorophyll fluorescence using fast repetition rate techniques: defining methodology and experimental protocols. Biochim Biophys Acta 1367:88-106

Lübben M, Morand K (1994) Novel prenylated hemes as cofactors of cytochrome oxidases: Archaea have modified heme- $a$ and heme- $o$. J Biol Chem 269:21473-21479

> Marchetti A, Parker MS, Moccia LP, Lin EO and others (2009) Ferritin is used for iron storage in bloom-forming marine pennate diatoms. Nature 457:467-470

> Masuda T, Takahashi S (2006) Chemiluminescent-based method for heme determination by reconstitution with horseradish peroxidase apo-enzyme. Anal Biochem 355: 307-309

> Measures CI, Landing WM, Brown MT, Buck CS (2008) High-resolution $\mathrm{Al}$ and $\mathrm{Fe}$ data from the Atlantic Ocean CLIVAR- $\mathrm{CO}_{2}$ repeat hydrography A16N transect: extensive linkages between atmospheric dust and upper ocean geochemistry. Global Biogeochem Cycles 22: GB1005, doi:10.1029/2007GB003042

> Mills MM, Ridame C, Davey M, LaRoche J, Geider RJ (2004) Iron and phosphorus co-limit nitrogen fixation in the eastern tropical North Atlantic. Nature 429:292-294

> Mochizuki N, Tanaka R, Grimm B, Masuda T and others (2010) The cell biology of tetrapyrroles: a life and death struggle. Trends Plant Sci 15:488-498

Moore CM, Mills MM, Milne A, Langlois R and others (2006) Iron limits primary productivity during spring bloom development in the central North Atlantic. Glob Change Biol 12:626-634

Patey MD, Achterberg EP, Rijkenberg MJA, Statham PJ, Mowlem M (2010) Interferences in the analysis of nanomolar concentrations of nitrate and phosphate in oceanic waters. Anal Chim Acta 673:109-116

> Pingree RD, Holligan PM, Mardell GT, Head RN (1976) The influence of physical stability on spring, summer and autumn phytoplankton blooms in the Celtic Sea. J Mar Biol Assoc UK 56:845-873

> Poulton AJ, Charalampopoulou A, Young JR, Tarran GA, Lucas MI, Quartly GD (2010) Coccolithophore dynamics in non-bloom conditions during late summer in the central Iceland Basin (July-August 2007). Limnol Oceanogr 55:1601-1613 
Raven JA (1990) Predictions of Mn and Fe use efficiencies of phototrophic growth as a function of light availability for growth and of C assimilation pathway. New Phytol 116: $1-18$

Richier S, Macey AI, Pratt NJ, Honey DJ, Moore CM, Bibby TS (2012) Abundances of iron-binding photosynthetic and nitrogen-fixing proteins of Trichodesmium both in culture and in situ from the north Atlantic. PLoS ONE 7: e35571

Rijkenberg MJA, Powell CF, Dall'Osto M, Nielsdottir MC and others (2008) Changes in iron speciation following a Saharan dust event in the tropical North Atlantic Ocean. Mar Chem 110:56-67

Rijkenberg MJA, Langlois RJ, Mills MM, Patey MD and others (2011) Environmental forcing of nitrogen fixation in the eastern tropical and sub-tropical north Atlantic Ocean. PLoS ONE 6:e28989

Rijkenberg MJA, Steigenberger S, Powell CF, van Haren $\mathrm{H}$, Patey MD, Baker AR, Achterberg EP (2012) Fluxes and distribution of dissolved iron the eastern (sub-) tropical North Atlantic Ocean. Global Biogeochem Cycles 26: GB3004, doi:10.1029/2011GB004264

> Robinson C, Poulton AJ, Holligan PM, Baker AR and others (2006) The Atlantic Meridional Transect (AMT) programme: a contextual view 1995-2005. Deep-Sea Res II 53:1485-1515

> Ross ON, Moore CM, Suggett DJ, MacIntyre HL, Geider RJ (2008) A model of photosynthesis and photo-protection based on reaction center damage and repair. Limnol Oceanogr 53:1835-1852

Ryan-Keogh TJ, Macey AI, Cockshutt AM, Moore CM, Bibby TS (2012) The cyanobacterial chlorophyll-bindingprotein isiA acts to increase the in vivo effective adsorption cross-section of PSI under iron limitation. J Phycol 48:145-154

Saito MA, Bertrand EM, Dutkiewicz S, Bulygin VV and others (2011) Iron conservation by reduction of metalloenzyme inventories in the marine diazotroph Crocosphaera watsonii. Proc Natl Acad Sci USA 108:2184-2189

Sanders R, Jickells T (2000) Total organic nutrients in Drake Passage. Deep-Sea Res I 47:997-1014

Seddon AM, Curnow P, Booth PJ (2004) Membrane proteins, lipids and detergents: not just a soap opera. Biochim Biophys Acta 1666:105-117

Sedwick PN, Church TM, Bowie AR, Marsay CM and others (2005) Iron in the Sargasso Sea (Bermuda Atlantic Timeseries Study region) during summer: Eolian imprint, spatiotemporal variability, and ecological implications. Global Biogeochem Cycles 19:GB4006, doi:10.1029/2004 GB002445

Sharples J, Moore CM, Hickman AE, Holligan PM, Tweddle JF, Palmer MR, Simpson JH (2009) Internal tidal mixing as a control on continental margin ecosystems. Geophys

Editorial responsibility: Steven Lohrenz,

New Bedford, Massachusetts, USA
Res Lett 36:L23603, doi:10.1029/2009GL040683

Stillman LC, Gassman ML (1978) Protoheme extraction from plant tissue. Anal Biochem 91:166-172

> Strzepek RF, Harrison PJ (2004) Photosynthetic architecture differs in coastal and oceanic diatoms. Nature 431: 689-692

> Suggett DJ, Le Floc'H E, Harris GN, Leonardos N, Geider RJ (2007) Different strategies of photoacclimation by two strains of Emiliania huxleyi (Haptophyta). J Phycol 43: 1209-1222

Sunda WG (2012) Feedback interactions between trace metal nutrients and phytoplankton in the ocean. Front Microbiol 3:204

Sunda WG, Huntsman SA (1995) Iron uptake and growth limitation in oceanic and coastal phytoplankton. Mar Chem 50:189-206

Sunda WG, Price NM, Morel FMM (2005) Trace metal ion buffers and their use in culture studies. In: Anderson RA (ed) Algal culturing techniques. Elsevier, Burlington, MA, p 35-65

Twining BS, Baines SB (2013) The trace metal composition of marine phytoplankton. Annu Rev Mar Sci 5:191-215

Twining BS, Baines SB, Fisher NS, Landry MR (2004) Cellular iron contents of plankton during the Southern Ocean Iron Experiment (SOFeX). Deep-Sea Res I 51:1827-1850

Ussher SJ, Worsfold PJ, Achterberg EP, Laes A, Blain S, Laan P, de Baar HJW (2007) Distribution and redox speciation of dissolved iron on the European continental margin. Limnol Oceanogr 52:2530-2539

> Vavilin DV, Vermaas WFJ (2002) Regulation of the tetrapyrrole biosynthetic pathway leading to heme and chlorophyll in plants and cyanobacteria. Physiol Plant 115:9-24

- Verardo DJ, Froelich PN, McIntyre A (1990) Determination of organic carbon and nitrogen in marine sediments using the Carlo-Erba NA 1500 analyzer. Deep-Sea Res A 37:157-165

Weber M, Prodohl A, Dreher C, Becker C and others (2011) SDS-facilitated in vitro formation of a transmembrane btype cytochrome is mediated by changes in local $\mathrm{pH}$. J Mol Biol 407:594-606

- Welschmeyer NA (1994) Fluorometric analysis of chlorophyll $a$ in the presence of chlorophyll $b$ and pheopigments. Limnol Oceanogr 39:1985-1992

Wilson WH, Carr NG, Mann NH (1996) The effect of phosphate status on the kinetics of cyanophage infection in the oceanic cyanobacterium Synechococcus sp. WH7803. J Phycol 32:506-516

- Wu J, Boyle E, Sunda W, Wen LS (2001) Soluble and colloidal iron in the oligotrophic North Atlantic and North Pacific. Science 293:847-849

Yu GH, Weinstein JD (1997) Heme synthesis and breakdown in isolated developing pea chloroplasts. Plant Physiol Biochem 35:223-234

Submitted: December 20, 2012; Accepted: April 10, 2013

Proofs received from author(s): May 15, 2013 